\title{
UWB system based on energy detection of derivatives of the Gaussian pulse
}

\author{
Song Cui and Fuqin Xiong
}

\begin{abstract}
A new method for energy detection ultra-wideband systems is proposed. The transmitter of this method uses two pulses that are different-order derivatives of the Gaussian pulse to transmit bit 0 or 1 . These pulses are appropriately chosen to separate their spectra in the frequency domain. The receiver is composed of two energydetection branches. Each branch has a filter which captures the signal energy of either bit 0 or 1 . The outputs of the two branches are subtracted from each other to generate the decision statistic. The value of this decision statistic is compared to the threshold to determine the transmitted bit. This new method has the same bit error rate (BER) performance as energy detection-based pulse position modulation (PPM) in additive white Gaussian noise channels. In multipath channels, its performance surpasses PPM and it also exhibits better BER performance in the presence of synchronization errors.
\end{abstract}

Keywords: ultra-wideband (UWB), energy detection, cross-modulation interference, synchronization error

\section{Introduction}

Ultra-wideband (UWB) impulse radio (IR) technology has become a popular research topic in wireless communications in recent years. It is a potential candidate for short-range, low-power wireless applications [1]. UWB systems convey information by transmitting subnanosecond pulses with a very low duty-cycle. These extremely short pulses produce fine time-resolution UWB signals in multipath channels, and this makes Rake receivers good candidates for UWB receivers. However, the implementation of Rake receivers is very challenging in UWB systems because Rake receivers need a large number of fingers to capture significant signal energy. This greatly increases the complexity of the receiver structure and the computational burden of channel estimation $[2,3]$. Rake receivers also need extremely accurate synchronization because of the use of correlators [3].

Due to the limitations in Rake receivers, many researchers shift their research to non-coherent UWB methods. As one of the conventional non-coherent technologies, energy detection (ED) has been applied to the field of UWB in recent years. Although ED is a sub-

\footnotetext{
* Correspondence: s.cui99@csuohio.edu

* Correspondence: s.cui99@csuohio.edu University, Cleveland, OH, USA
}

optimal method, it has many advantages over coherent receivers. It does not use correlator at the receiver, so channel estimation is not required. Unlike Rake receivers, the receiver structure of ED is very simple $[2,4]$. Also ED receivers do not need as accurate synchronization as Rake receivers. ED has been applied to on-off keying (OOK) and pulse position modulation (PPM) [5].

In this article, a new method to realize ED UWB system is proposed. In this method, two different-order derivatives of the Gaussian pulse are used to transmit bit 1 or 0 . This pair of pulses is picked appropriately to separate the spectra of the pulses in the frequency domain. This separation of spectra is similar to that of frequency shift keying (FSK) in continuous waveform systems. In UWB systems, no carrier modulation is used, and the signals are transmitted in baseband. The popular modulation methods are PPM and pulse amplitude modulation (PAM), which achieve modulation by changing the position or amplitude of the pulse. But our method is different to PPM and PAM. The modulation is achieved using two different-order derivatives of the Gaussian pulse, which occupy different frequency ranges. Our method still does not involve carrier modulation and the signal is still transmitted in baseband like other UWB systems. We call this new method as the Gaussian FSK (GFSK) UWB. Although some previous 
studies about FSK-UWB have been proposed in [6-8], but these methods all use sinusoidal waveforms as carriers to modulate signal spectra to desired locations. In UWB systems, the transmission of the signal is carrierless, so it needs fewer RF components than carrierbased transmission. This makes UWB transceiver structure much simpler and cheaper than carrier-based systems. Without using carrier modulation, the mixer and local oscillator are removed from the transceiver. This greatly reduces the complexity and cost, especially when a signal is transmitted in high frequency. Carrier recover stage is also removed from the receiver [9]. It seems that these FSK-UWB methods proposed by previous researchers are not good methods since they induce carrier modulation. In recent years, pulse shape modulation (PSM) is also proposed for UWB systems. This modulation method uses orthogonal pulse waveform to transmit different signals. Hermite and modified Hermite pulses are chosen as orthogonal pulses in PSM method. However, Hermit pulse is not suitable to our GFSK system. Although different-order Hermit pulses are orthogonal, their spectra are not well separated as differentorder Gaussian pulses. In $[10,11]$, the spectra of different-order Hermite pulses greatly overlapped, and in [12] the spectra of some Hermite pulses with different-order almost entirely overlapped together. Since the ED receiver exploits the filter to remove out of band energy and capture the signal energy, Hermite pulse is not a good candidate since the overlapped spectra of different-order pulses cannot be distinguished by the filters. In Gaussian pulse family, the bandwidths of different-order pulses are similar. However, the center frequencies are greatly different. The center frequency of a higher-order pulse is located at higher frequency location [13]. When an appropriate pulse pair is chosen, the signal spectra will effectively be separated. We can use two filters, which have different passband frequency ranges, to distinguish the different signals effectively. This is the reason we chose Gaussian pulse in this article.

The research results show that our GFSK system has the same bit error rate (BER) performance as an ED PPM system in additive white Gaussian noise (AWGN) channels. In multipath channels, GFSK does not suffer cross-modulation interference as in PPM, and the BER performance greatly surpasses that of PPM. Also this method is much more immune to synchronization errors than PPM.

The rest of the article is structured as follows. Section 2 introduces the system models. Section 3 evaluates system performance in AWGN channels. Section 4 evaluates system performance in multipath channels. The effect of synchronization errors on system performance is analyzed in Section 5. In Section 6, the numerical results are analyzed. In Section 7, the conclusions are stated.

\section{System models}

\subsection{System model of GFSK}

The design idea of this new system originates from spectral characteristics of the derivatives of the Gaussian pulse. The Fourier transform $X_{\mathrm{f}}$ and center frequency $f_{\mathrm{c}}$ of the $k$ th-order derivative are given by [13]

$$
\begin{aligned}
& X_{\mathrm{f}} \propto f^{k} \exp \left(-\pi f^{2} \alpha^{2} / 2\right) \\
& f_{\mathrm{C}}=\sqrt{k} /(\alpha \sqrt{\pi})
\end{aligned}
$$

where $k$ is the order of the derivative and $f$ is the frequency. The pulse shaping factor is denoted by $\alpha$. If we assign a constant value to $\alpha$ and change the $k$ value in (1), we obtain spectral curves for different-order derivatives. It is surprising to find that those curves have similar shapes and bandwidths. The major difference is their center frequencies. The reason that the change of center frequencies can be explained directly from (2). If the values of $k$ and $\alpha$ are appropriately chosen, it is always possible to separate the spectra of the two pulses. To satisfy the UWB emission mask set by Federal Communications Commission (FCC), we chose the pulse-pair for analysis and simulation in this article as follows: the two pulses are 10th- and 30th-order derivatives of the Gaussian pulse, respectively, and the shape factor is $\alpha=$ $0.365 \times 10^{-9}$. In Figure 1, the power spectrum density (PSD) of the two pulses and FCC emission mask are shown. A simple method to plot the PSD of two pulses is to plot $\left|X_{f}\right|^{2}$ and set the peak value of $\left|X_{f}\right|^{2}$ to -41.3 $\mathrm{dBm}$, which is the maximum power value of FCC emission mask. From Figure 1, we can see that both the PSD of two pulses satisfy the FCC mask. However, we should not get confused about the spectral separation of these two pulses. The overlapped section of the signal spectra include very low signal energy, and the only reason to affect our observation is that PSD of pulses and FCC mask in Figure 1 are plotted using logarithmic scale. A linear scale version of Figure 1 is shown in Figure 2. In Figure 2, the peak value of signal spectra and FCC mask is normalized to 1 , it dose not mean the absolute transmitting power is 1 . From Figure 2, it is clearly seen that intersection point of the spectral curves is lower than 0.1 , which denotes the $-10 \mathrm{~dB}$ power point. So the overlapped part of signal spectra include very low energy, and the spectra of these two pulses are effectively separated.

Exploiting the spectral characteristics of the pulses, we will construct the transmitter of our GFSK system. Without loss of generality, we focus on single user 


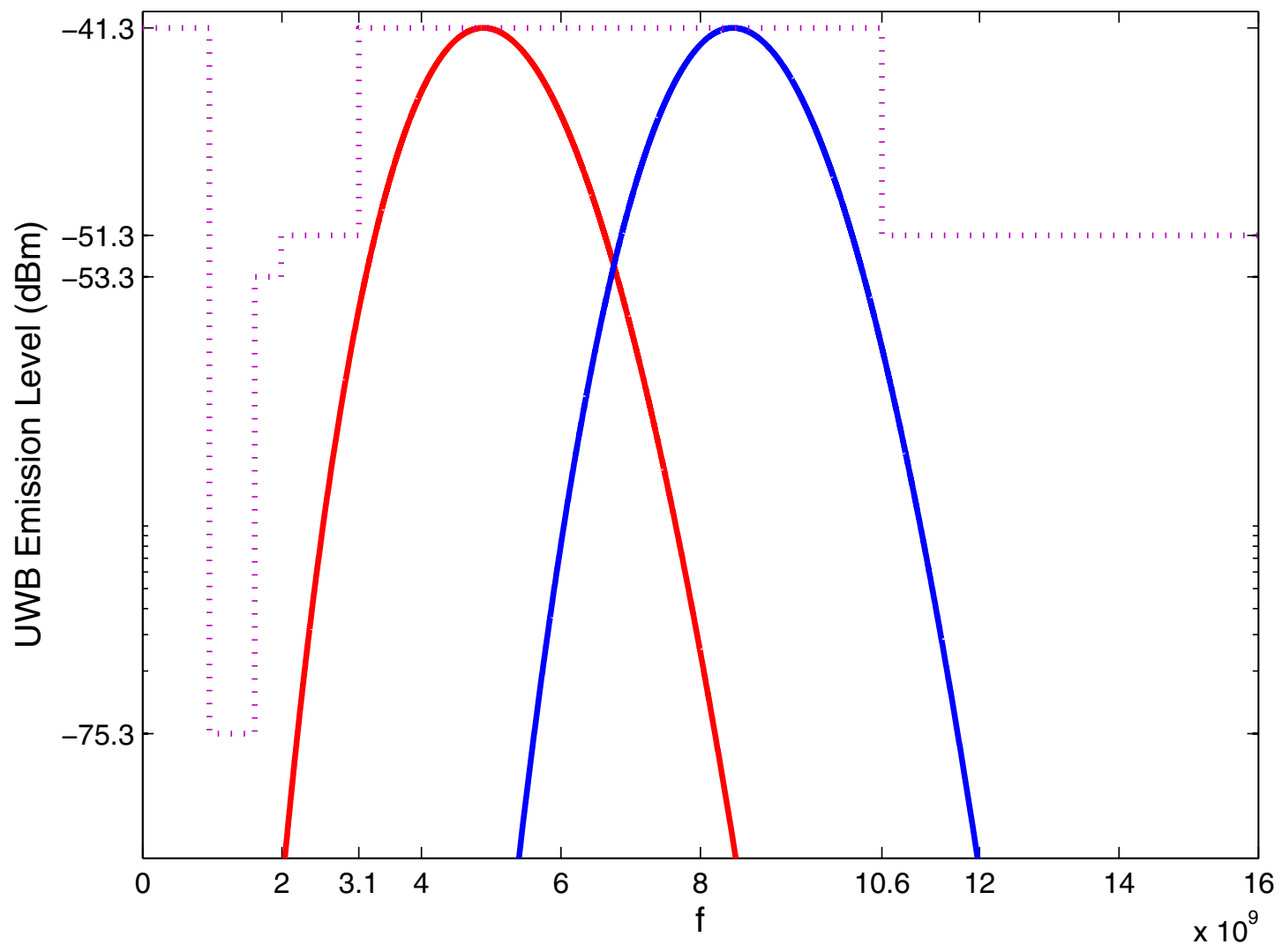

Figure 1 PSD of pulses versus FCC emission mask (logarithmic scale).

communication case in this article, and a bit is transmitted only once. The transmitted signal of this system is

$$
s(t)_{\mathrm{GFSK}}=\sum_{j} \sqrt{E_{p}}\left(b_{j} p_{1}\left(t-j T_{f}\right)+\left(1-b_{j}\right) p_{2}\left(t-j T_{\mathrm{f}}\right)\right)
$$

where $p_{1}(t)$ and $p_{2}(t)$ denote the pulse waveforms of different-order derivatives with normalized energy, and $E_{p}$ is the signal energy. The $j$ th transmitted bit is denoted by $b_{j}$. The frame period is denoted by $T_{f}$. The modulation is carried out as follows: when bit 1 is transmitted, the value of $b_{j}$ and $1-b_{j}$ are 1 and 0 , respectively, so $p_{1}(t)$ is transmitted. Similarly, the transmitted waveform for bit 0 is $p_{2}(t)$.

The receiver is depicted in Figure 3. It is separated into two branches, and each branch is a conventional energy detection receiver. The only difference between the two branches is the passband frequency ranges of filters. Filter 1 is designed to pass the signal energy of $p_{1}$ $(t)$ and reject that of $p_{2}(t)$, and Filter 2 passes the signal energy of $p_{2}(t)$ and rejects that of $p_{1}(t)$. The signal arriving at the receiver is denoted by $s(t)$, the AWGN is denoted by $n(t)$, and the sum of $s(t)$ and $n(t)$ is denoted by $r(t)$. The integration interval is $T \leq T_{\mathrm{f}}$. The decision statistic is given by $Z=Z_{1}-Z_{2}$, where $Z_{1}$ and $Z_{2}$ are the outputs of branches 1 and 2 , respectively. Finally, $Z$ is compared with threshold $\gamma$ to determine the transmitted bit. If $Z \geq \gamma$, then the transmitted bit is 1 , otherwise it is 0 .

In this GFSK system, the appropriate pulse pair is not limited to the 10th- and 30th-order in Figure 1, and the choice of the pulse pair depends on the bandwidth requirement of the system and its allocated frequency range. Increasing the value of $\alpha$ decreases the bandwidth, and the center frequencies of the pulses can be shifted to higher frequencies by increasing the order of the derivatives [13]. Also, the spectral separation of a pulse pair can be increased by increasing the difference of the orders of the derivatives. Although the implementation of this system needs high-order derivatives, it is already feasible using current technology to generate such pulses. Many articles describing the hardware implementation of pulse generators for high-order derivatives have been published. In [14], a 7 th-order pulse 


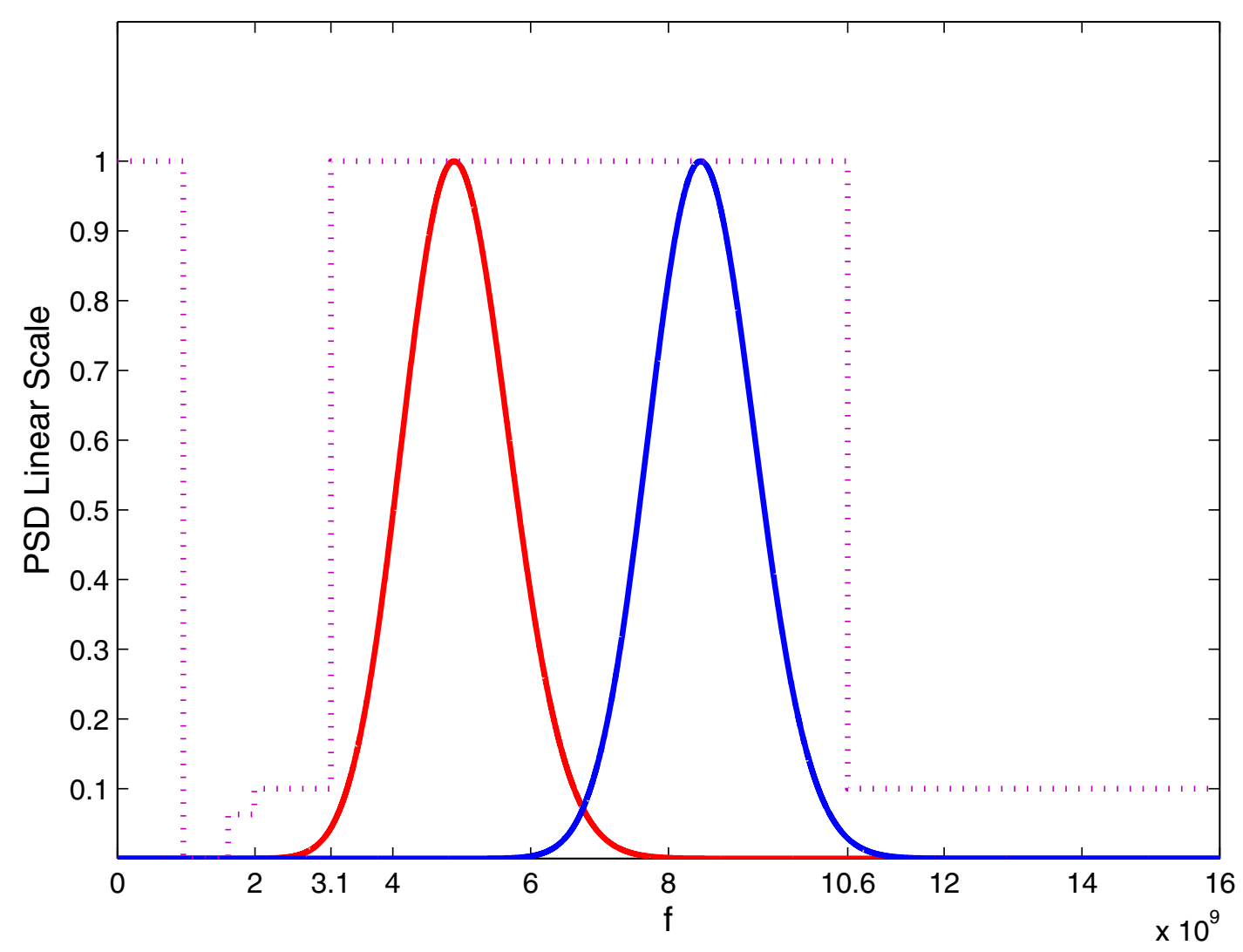

Figure 2 PSD of pulses versus FCC emission mask (linear scale).

generator is proposed, and the generator in [15] is capable of producing a 13th-order pulse. In [16], the center frequency of the generated pulse is $34 \mathrm{GHz}$.

In this article, the performance of this new system is compared to existing systems, and the models of these systems are simply described as follows. When the transmitted data is 0 , the OOK system does not transmit a signal, so it has difficulty to achieve synchronization, especially when a stream of zeros is transmitted [9]. Therefore, it is not compared in this article.

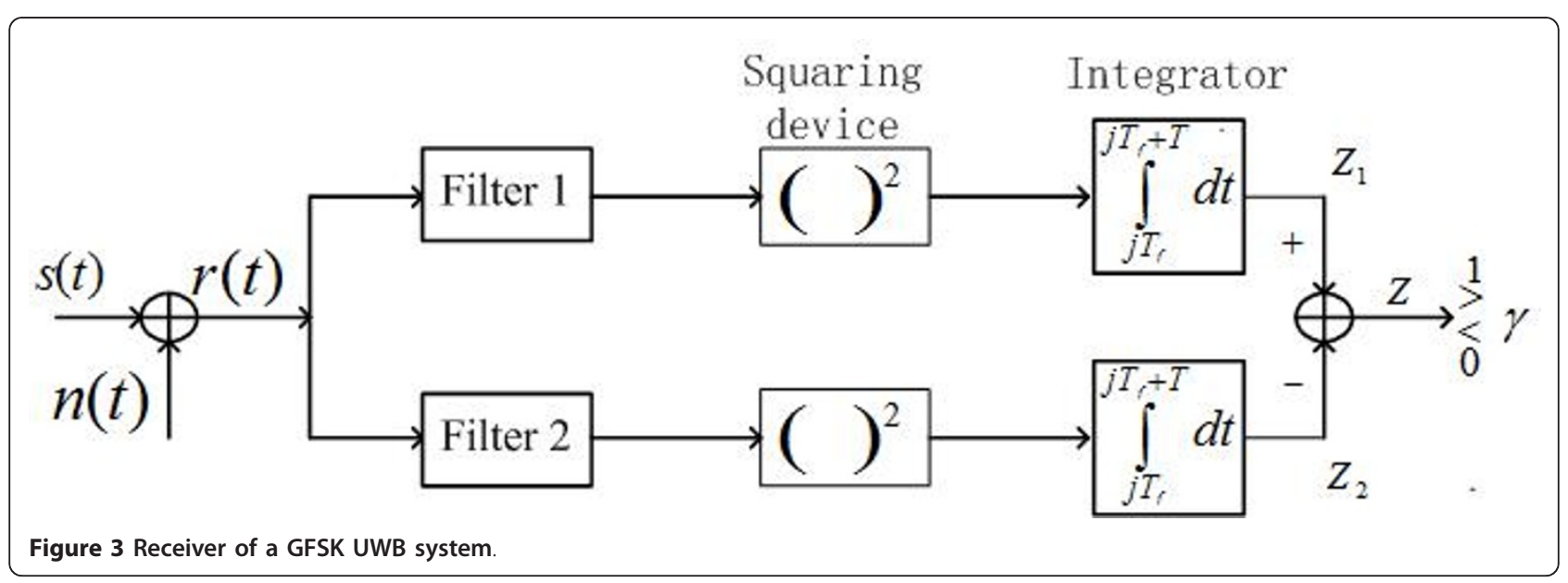




\subsection{System model of PPM}

The transmitted signal of a PPM system is [1]

$$
s(t)_{\mathrm{PPM}}=\sum_{j} \sqrt{E_{p}} p\left(t-j T_{\mathrm{f}}-\delta b_{j}\right)
$$

where $\delta$ is called the modulation index, and the pulse shift amount is determined by $\delta b_{j}$. Other parameters have the same meaning as (3). At the receiver, after the received signal pass through a square-law device and an integrator, the decision statistic $Z$ is obtained as [17]

$$
Z=Z_{1}-Z_{2}=\int_{j T_{f}}^{j T_{f}+T} r^{2}(t) d t-\int_{j T_{f}+\delta}^{j T_{f}+\delta+T} r^{2}(t) d t
$$

where $T \leq \delta$ denotes the length of integration interval. The decision threshold of PPM is $\gamma=0$. If $Z \geq \gamma=0$, the transmitted bit is 0 , otherwise it is 1 .

\subsection{Parameters}

Some parameter values are given below, and these values are used later in simulation. We can use the symbolic calculation tool MAPLE to perform $\frac{d^{10}}{d t^{10}}\left(\frac{\sqrt{2}}{\alpha} e^{\frac{-2 \pi t^{2}}{\alpha^{2}}}\right)$ and $\frac{d^{30}}{d t^{30}}\left(\frac{\sqrt{2}}{\alpha} e^{\frac{-2 \pi t^{2}}{\alpha^{2}}}\right)$ to obtain the 10th- and 30th-order derivatives, where $\frac{\sqrt{2}}{\alpha} e^{\frac{-2 \pi t^{2}}{\alpha^{2}}}$ is the Gaussian pulse [13].

The equations for the 10th- and 30th-order derivatives are obtained as follows:

$$
\begin{aligned}
& s(t)_{10}=\left(-967680+\frac{19353600 \pi t^{2}}{\alpha^{2}}\right. \\
& -\frac{51609600 \pi^{2} t^{4}}{\alpha^{4}}+\frac{41287680 \pi^{3} t^{6}}{\alpha^{6}} \\
& \left.-\frac{11796480 \pi^{4} t^{8}}{\alpha^{8}}+\frac{1048576 \pi^{5} t^{10}}{\alpha^{10}}\right) e^{\frac{-2 \pi t^{2}}{\alpha^{2}}} \\
& s(t)_{30}=\left(-6646766139202842132480000+\frac{398805968352170527948800000 \pi t^{2}}{\alpha^{2}}\right. \\
& -\frac{3722189037953591594188800000 \pi^{2} t^{4}}{\alpha^{4}}+\frac{12903588664905784193187840000 \pi^{3} t^{6}}{\alpha^{6}} \\
& -\frac{22120437711267058616893440000 \pi^{4} t^{8}}{\alpha^{8}}+\frac{21628872428794457314295808000 \pi^{5} t^{10}}{\alpha^{10}} \\
& -\frac{13108407532602701402603520000 \pi^{6} t^{12}}{\alpha^{12}}+\frac{5185743639271398357073920000 \pi^{7} t^{14}}{\alpha^{14}} \\
& -\frac{1382864970472372895219712000 \pi^{8} t^{16}}{\alpha^{16}}+\frac{253073327929584582131712000 \pi^{9} t^{18}}{\alpha^{18}} \\
& -\frac{31967157212158052479795200 \pi^{10} t^{20}}{\alpha^{20}}+\frac{2767719239147883331584000 \pi^{11} t^{22}}{\alpha^{22}} \\
& -\frac{160447492124514975744000 \pi^{12} t^{24}}{\alpha^{24}}+\frac{5924215093828245258240 \pi^{13} t^{26}}{\alpha^{26}} \\
& \left.-\frac{125380213625994608640 \pi^{14} t^{28}}{\alpha^{28}}+\frac{1152921504606846976 \pi^{15} t^{30}}{\alpha^{30}}\right) e^{\frac{-2 \pi t^{2}}{\alpha^{2}}}
\end{aligned}
$$

where (6) and (7) are the equations of the 10th- and 30th-order derivatives of the Gaussian pulse. These two equations are the simplified versions of the original ones obtained from MAPLE. The common factors of the terms in parentheses of (6) and (7) are $\frac{\sqrt{2} \pi^{5}}{\alpha^{11}}$ and $\frac{\sqrt{2} \pi^{15}}{\alpha^{31}}$, respectively. They are constants and do not affect the waveform shapes, so they been removed to simplify equations. The value of $\alpha$ is set to $0.365 \times 10^{-9}$ and the width of the pulses are chosen to be $2.4 \alpha=0.876 \times 10^{-9}$ $=0.876 \mathrm{~ns}$ (the detailed method to choose pulse width for a shaping factor $\alpha$ can be found from Benedetto and Giancola [13]). For GFSK, we use the 10th-order derivative to transmit bit 1 , and the 30th-order to transmit bit 0 . For PPM, we use the 10th-order derivative.

\section{BER performance in AWGN channels}

\subsection{BER performance of GFSK in AWGN channels}

In Figure $3, Z_{1}$ and $Z_{2}$ are the outputs of conventional energy detectors, and they are defined as chi-square variables with approximately a degree of $2 T W$ [18], where $T$ is the integration time and $W$ is the bandwidth of the filtered signal. A popular method for energy detection, called Gaussian approximation, has been developed to simplify the derivation of the BER formula. When $2 T W$ is large enough, a chi-square variable can be approximated as a Gaussian variable. This method is commonly used in energy detection communication systems $[5,17,19,20]$. The mean value and variance of this approximated Gaussian variable are [21]

$$
\begin{aligned}
& \mu=N_{0} T W+E \\
& \sigma^{2}=N_{0}^{2} T W+2 N_{0} E
\end{aligned}
$$

where $\mu$ and $\sigma^{2}$ are the mean value and variance, respectively. The double-sided power spectral density of AWGN is $N_{0} / 2$, where $N_{0}$ is the single-sided power spectral density. The signal energy which passes through the filter is denoted by $E$. If the filter rejects all of the signal energy, then $E=0$. In Figure 3, when bit 1 is transmitted, the signal energy passes through Filter 1 and is rejected by Filter 2 . The probability density function (pdf) of $Z_{1}$ and $Z_{2}$ can be expressed as $Z_{1} \sim N\left(N_{0} T W+E_{b}, N_{0}^{2} T W+2 N_{0} E_{b}\right) \quad$ and $Z_{2} \sim N\left(N_{0} T W, N_{0}^{2} T W\right)$, where $E_{b}$ denotes the bit energy. In this article, the same bit is not transmitted repeatedly, so $E_{b}$ is used to replace $E$ here. Since $Z=Z_{1}$ - $Z_{2}$, the pdf of $Z$ is

$$
H_{1}: Z \sim N\left(E_{b}, 2 N_{0}^{2} T W+2 N_{0} E_{b}\right)
$$

Using the same method, the pdf of $Z$ when bit 0 is transmitted is

$$
H_{0}: Z \sim N\left(-E_{b}, 2 N_{0}^{2} T W+2 N_{0} E_{b}\right)
$$

After obtaining the pdf of $Z$, we follow the method given in [19] to derive the BER formula. First, we calculate the BER when bits 0 and 1 are transmitted as follows: 


$$
\begin{aligned}
& P_{0}=\int_{\gamma}^{\infty} f_{0}(x) d x=\int_{\gamma}^{\infty} \frac{1}{\sqrt{2 \pi} \sigma_{0}} e^{-\frac{\left(x-\mu_{0}\right)^{2}}{2 \sigma_{0}^{2}}} d x \\
& P_{1}=\int_{-\infty}^{\gamma} f_{1}(x) d x=\int_{-\infty}^{\gamma} \frac{1}{\sqrt{2 \pi} \sigma_{1}} e^{-\frac{\left(x-\mu_{1}\right)^{2}}{2 \sigma_{1}^{2}}} d x
\end{aligned}
$$

where $f_{0}(x)$ and $f_{1}(x)$ denote the probability density functions, and $\gamma$ denotes the decision threshold. From (10) and (11), it is straightforward to obtain $\mu_{0}=E_{b}$, $\sigma_{0}^{2}=2 N_{0}^{2} T W+2 N_{0} E_{b}, \mu_{1}=E_{b}, \sigma_{1}^{2}=2 N_{0}^{2} T W+2 N_{0} E_{b}$. Substituting these parameter values into (12) and (13), and then expressing $P_{0}$ and $P_{1}$ in terms of the complementary error function $Q(\cdot)$, we obtain

$$
\begin{aligned}
& P_{0}=Q\left(\left(E_{b}+\gamma\right) / \sqrt{2 N_{0}^{2} T W+2 N_{0} E_{b}}\right) \\
& P_{1}=Q\left(\left(E_{b}-\gamma\right) / \sqrt{2 N_{0}^{2} T W+2 N_{0} E_{b}}\right)
\end{aligned}
$$
$[5,19]$

The optimal threshold is obtained by setting $P_{0}=P_{1}$

$$
\left(E_{b}+\gamma\right) / \sqrt{2 N_{0}^{2} T W+2 N_{0} E_{b}}=\left(E_{b}-\gamma\right) / \sqrt{2 N_{0}^{2} T W+2 N_{0} E_{b}}
$$

Solving equation (16), the optimal threshold is obtained as

$$
\gamma=0
$$

The total BER is $P_{e}=0.5\left(P_{0}+P_{1}\right)$. Since $P_{0}=P_{1}$, it follows that $P_{e}=P_{0}$. Substituting (17) into (14), the total BER of GSFK in AWGN channels is

$$
P_{e}=Q\left(\frac{E_{b} / N_{0}}{\sqrt{2 T W+2 E_{b} / N_{0}}}\right)
$$

\subsection{PPM in AWGN channels}

The BER equation of ED PPM has been derived in [5]. It has the same BER performance as GFSK systems. So (18) is valid for both GFSK and PPM systems.

\section{BER performance in multipath channels}

In this section, the BER performances of PPM and GFSK in multipath channels are researched. The channel model of the IEEE 802.15.4a standard [22] is used in this article. After the signal travels through a multipath channel, it is convolved with the channel impulse response. The received signal becomes

$$
r(t)=s(t) \otimes h(t)+n(t)
$$

where $h(t)$ denotes the channel impulse response and $n(t)$ is AWGN. The symbol $\otimes$ denotes the convolution operation. The IEEE 802.15.4a model is an extension of the Saleh-Valenzeula (S-V) model. The channel impulse response is

$$
h(t)=\sum_{l=0}^{L} \sum_{k=0}^{K} \alpha_{k, l} \exp \left(j \phi_{k, l}\right) \delta\left(t-T_{l}-\tau_{k, l}\right)
$$

where $\delta(t)$ is Dirac delta function, and $\alpha_{k, l}$ is the tap weight of the $k$ th component in the $l$ th cluster. The delay of the $l$ th cluster is denoted by $T_{l}$ and $\tau_{k, l}$ is the delay of the $k$ th multipath component relative to $T_{l}$. The phase $\varphi_{k, l}$ is uniformly distributed in the range $[0,2 \pi]$.

\subsection{PPM in multipath channels}

In PPM systems, the modulation index $\delta$ in (4) must be chosen appropriately. If it is designed to be less than the maximum channel spread $D$, the cross-modulation interference (CMI) will occur $[17,20,23]$. When CMI occurs, the system performance will be degraded greatly. Even increasing the transmitting power will not improve the performance because of the proportional increase of interference [23]. The effect of $\delta$ on BER performance of PPM has been analyzed in [20]. But the BER equation in [20] is not expressed with respect to $E_{b} / N_{0}$. For convenience in the following analysis, the BER equation will be expressed in terms of $E_{b} / N_{0}$ in this article. Figure 4 is the frame structures of PPM in the presence of CMI.

The relationship of $\delta$ with $T_{0}$ and $T_{1}$ is set to $\delta=T_{0}=$ $T_{1}$ as in [17], and $T_{0}$ and $T_{1}$ are the time intervals reserved for multipath components of bits 0 and 1 , respectively.

Synchronization is assumed to be perfect here. When $\delta$ is less than the maximum channel spread $D$, some multipath components of bit 0 fall into the interval $T_{1}$, and therefore CMI occurs. But the multipath components of bit 1 do not cause CMI. Some of them fall into the guard interval $T_{g}$, which is designed to prevent inter-frame interference (IFI). The frame period is $T_{f}=$ $T_{0}+T_{1}+T_{g}$. If $T_{g}$ is chosen to be too large, it will waste transmission time. So we follow the method in [17] and set $T_{f}=\delta+D$. This will always achieve as high a data rate as possible without inducing IFI. And the integration time is set to $T=T_{0}=T_{1}=\delta$ [17] in this article.

When bit 0 is transmitted, the pdfs of $Z_{1}$ and $Z_{2}$ are

$$
Z_{1} \sim N\left(N_{0} T W+\beta_{a} E_{b}, N_{0}^{2} T W+2 N_{0} \beta_{a} E_{b}\right), Z_{2} \sim N\left(N_{0} T W+\beta_{b} E_{b}, N_{0}^{2} T W+2 N_{0} \beta_{b} E_{b}\right) .
$$

Since $Z=Z_{1}-Z_{2}$, we have

$$
H_{0:} Z \sim N\left(\left(\beta_{a}-\beta_{b}\right) E_{b}, 2 N_{0}^{2} T W+2 N_{0}\left(\beta_{a}+\beta_{b}\right) E_{b}\right)(21)
$$



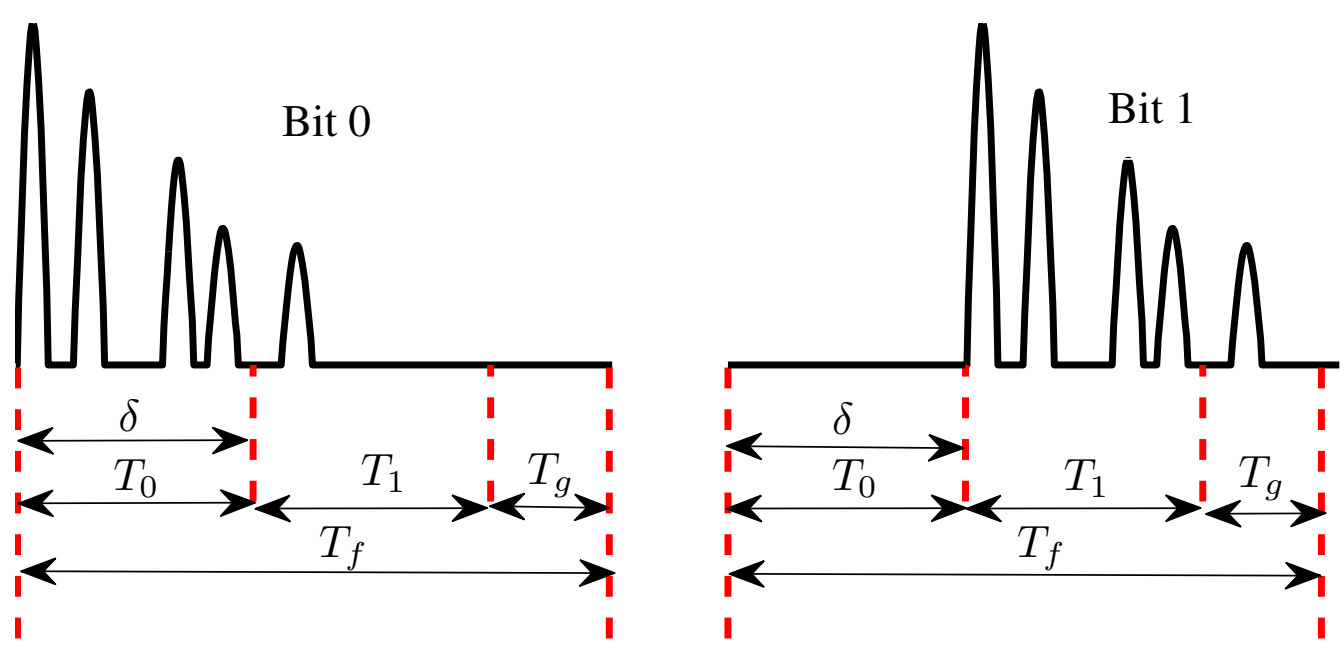

Figure 4 PPM frame structures in multipath channels

where $\beta_{a}=E_{T_{0}} / E_{b}$ and $\beta_{b}=E_{T_{1}} / E_{b}$. The meanings of $E_{T_{0}}$ and $E_{T_{1}}$ are the captured signal energies in integration interval $T_{0}$ and $T_{1}$, respectively. The values of $\beta_{a}$ and $\beta_{b}$ are in the range $[0,1]$. When bit 1 is transmitted, $E_{T_{0}}=0$, the pdfs become $Z_{1} \sim N\left(N_{0} T W, N_{0}^{2} T W\right)$ and $Z_{2} \sim N\left(N_{0} T W+\beta_{a} E_{b}, N_{0}^{2} T W+2 N_{0} \beta_{a} E_{b}\right)$. The pdf of $Z$ is

$$
H_{1:} Z \sim N\left(-\beta_{a} E_{b}, 2 N_{0}^{2} T W+2 N_{0} \beta_{a} E_{b}\right)
$$

where the $\beta_{a}$ in (22) has the same value as that in (21), but their meaning are different. In (22), $\beta_{a}=E_{T_{1}} / E_{b}$. Since the threshold is $\gamma=0$, the BER formula of PPM is

$$
P_{e}=0.5 \int_{-\infty}^{0} f_{0}(x) d x+0.5 \int_{0}^{\infty} f_{1}(x) d x
$$

where $f_{0}(x)$ and $f_{1}(x)$ are the pdfs corresponding to (21) and (22). Therefore, the BER is

$$
P_{e}=\frac{1}{2} Q\left(\frac{\left(\beta_{a}-\beta_{b}\right)\left(E_{b} / N_{0}\right)}{\sqrt{2 T W+2\left(\beta_{a}+\beta_{b}\right)\left(E_{b} / N_{0}\right)}}\right)+\frac{1}{2} Q\left(\frac{\beta_{a}\left(E_{b} / N_{0}\right)}{\sqrt{2 T W+2 \beta_{a}\left(E_{b} / N_{0}\right)}}\right)
$$

When there is no CMI, $\beta_{a}=1$ and $\beta_{b}=0$, (24) reduces to (18).

\subsection{GFSK in multipath channels}

Figure 5 is the frame structure of GFSK in multipath channels. CMI does not occur in GFSK systems as it does in PPM systems. In order to compare GFSK to

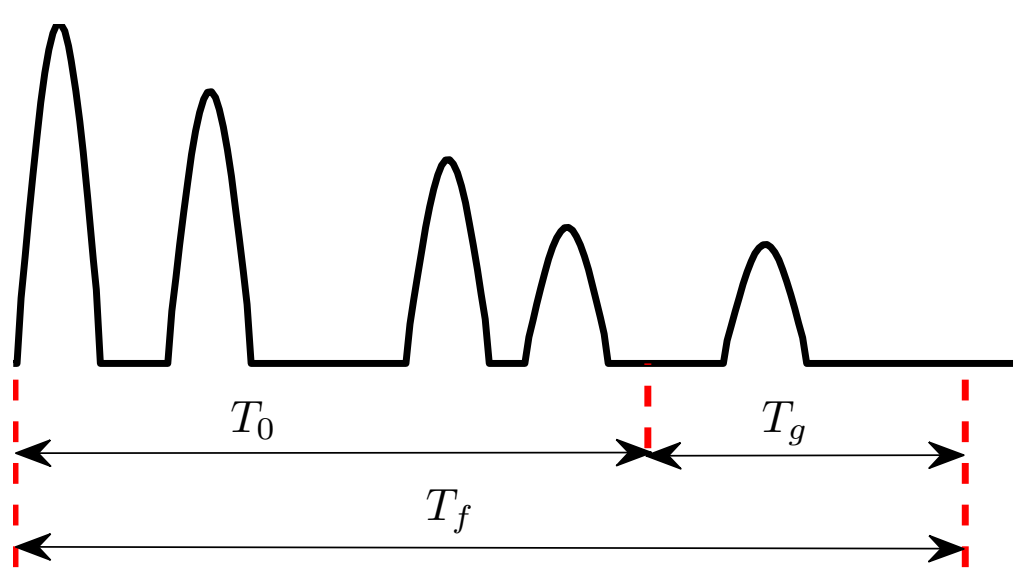

Figure 5 A GFSK frame structure in multipath channels 
PPM under the same energy capture condition, the integration interval $T_{0}$ of GFSK has the same length as the $T_{0}$ of PPM. Also synchronization is assumed to be perfect as in PPM. The guard interval is $T_{\mathrm{g}}$, and the frame period is set to $T_{\mathrm{f}}=T_{0}+T_{\mathrm{g}}=D$. This will achieve the maximum data rate and prevent IFI simultaneously. This frame structure is applied to both bits 0 and 1 . From Figure 5, it is straightforward to obtain the pdfs of $Z$ when bits 1 and 0 are transmitted as follows:

$$
\begin{aligned}
& H_{1}: Z \sim N\left(\lambda E_{b}, 2 N_{0}^{2} T W+2 N_{0} \lambda E_{b}\right) \\
& H_{0}: Z \sim N\left(-\lambda E_{b}, 2 N_{0}^{2} T W+2 N_{0} \lambda E_{b}\right)
\end{aligned}
$$

where $\lambda=E_{T_{0}} / E_{b}$. Using (12) and (13), and following the method in Section 3.1, we obtain the decision threshold and BER

$$
\begin{aligned}
& \gamma=0 \\
& P_{e}=Q\left(\frac{\lambda E_{b} / N_{0}}{\sqrt{2 T W+2 \lambda E_{b} / N_{0}}}\right)
\end{aligned}
$$

The channel model of IEEE 802.15.4a does not consider the antenna effect [22], so we do not add the antenna effect into our analysis. Also the frequency selectivity is not considered in analysis. If antenna and frequency selectivity are considered, the path loss of signals for bits 1 and 0 are different. So the energies of bits 1 and 0 are different at the receiver side. The threshold will not be 0 and the BER equation also will be different to (28). Because different antenna has different effect to signals, and frequency selectivity depends on the location of center frequency and signal bandwidth, we do not consider these two factors in the derivation of (28).

\section{Performance analysis in the presence of synchronization errors}

5.1 PPM performance in the presence of synchronization errors

Figure 6 depicts the PPM frame structures when synchronization errors $\varepsilon$ occur. The modulation index is set to $\delta=D=T_{0}=T_{1}$, so no CMI occurs. Assuming that coarse synchronization has been achieved, the BER performance of PPM and GFSK are compared in the range $\varepsilon \in[0, D / 2]$. To prevent IFI, the frame length is set to $T_{\mathrm{f}}=2 D+T_{\mathrm{g}}$, where the guard interval $T_{\mathrm{g}}$ equals to $D /$ 2 , the maximum synchronization error used in this article. When bit 0 is transmitted, we have $Z_{1} \sim N\left(N_{0} T W+\eta E_{b}, N_{0}^{2} T W+2 \eta E_{b} N_{0}\right) \quad$ and $Z_{2} \sim N\left(N_{0} T W, N_{0}^{2} T W\right)$. The pdf of $Z$ is

$$
H_{0}: Z \sim N\left(\eta E_{b}, 2 N_{0}^{2} T W+2 \eta E_{b} N_{0}\right)
$$

where $\eta=E_{T_{0}} / E_{b}$, and $E_{T_{1}}=0$. When bit 1 is transmitted, we have $Z_{1} \sim N\left(N_{0} T W+(1-\eta) E_{b}, N_{0}^{2} T W+2(1-\eta) E_{b} N_{0}\right)$ and $Z_{2} \sim N\left(N_{0} T W+\eta E_{b}, N_{0}^{2} T W+2 \eta E_{b} N_{0}\right)$. And then we obtain

$$
H_{1}: Z \sim N\left((1-2 \eta) E_{b}, 2 N_{0}^{2} T W+2 E_{b} N_{0}\right)
$$

where $\eta$ in (30) has the same value as that in (29), but in (30), $\eta=E_{T_{1}} / E_{b}$, and $E_{T_{0}}=(1-\eta) E_{b}$. Using (23), the
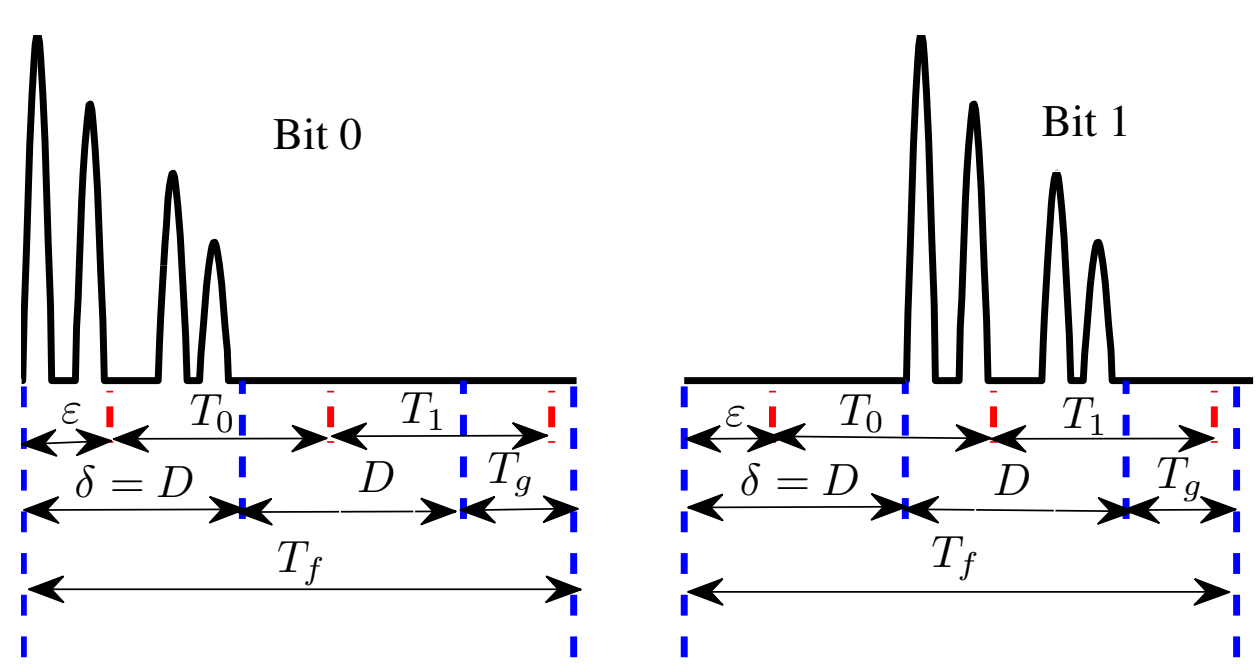

Figure 6 PPM frame structures in the presence of synchronization errors. 
total BER is

$$
P_{e}=\frac{1}{2} Q\left(\frac{\eta E_{b} / N_{0}}{\sqrt{2 T W+2 \eta E_{b} / N_{0}}}\right)+\frac{1}{2} Q\left(\frac{(2 \eta-1) E_{b} / N_{0}}{\sqrt{2 T W+2 E_{b} / N_{0}}}\right)
$$

\subsection{GFSK performance in the presence of synchronization} errors

Figure 7 depicts the GFSK frame structure in the presence of synchronization errors. The integration interval $T_{0}=D$ is the same as that of PPM. The frame length is $T_{\mathrm{f}}=T_{\mathrm{g}}+D$, where $T_{\mathrm{g}}=D / 2$ as in Section 5.1. From Figure 7 , the pdfs of $Z$ are

$$
\begin{aligned}
& H_{1}: Z \sim N\left(\rho E_{b}, 2 N_{0}^{2} T W+2 N_{0} \rho E_{b}\right) \\
& H_{0}: Z \sim N\left(-\rho E_{b}, 2 N_{0}^{2} T W+2 N_{0} \rho E_{b}\right)
\end{aligned}
$$

where $\rho=E_{T_{0}} / E_{b}$. Using (12) and (13), and following the method in Section 3.1, the decision threshold, and total BER are

$$
\begin{aligned}
& \gamma=0 \\
& P_{e}=Q\left(\frac{\rho E_{b} / N_{0}}{\sqrt{2 T W+2 \rho E_{b} / N_{0}}}\right)
\end{aligned}
$$

As in Section 4.2, we do not consider the effects of antenna and frequency selectivity in analysis.

\section{Numerical results and analysis}

Figure 8 shows the BER curves of GFSK systems in AWGN channels. In simulation, the bandwidth of the filters is $3.52 \mathrm{GHz}$, and the pulse duration is $0.876 \mathrm{~ns}$. Analytical BER curves are obtained directly from (18). When $2 T W$ is increased, there is a better match between the simulated and analytical curves, because the Gaussian approximation is more accurate under large $2 T W$ values [19]. After the bandwidth $W$ is chosen, the only way to change $2 T W$ is to change the length of integration time $T$. Therefore, when $T$ is increased, the Gaussian approximation is more accurate. However, increasing $T$ degrades BER performance because more noise energy is captured. When an UWB signal passes through a multipath channel, the large number of multipath components result in a very long channel delay. In order to capture the effective signal energy, the integration interval must be very long. This is why Gaussian approximation is commonly used in UWB systems. In the following, we will compare the BER performance of GFSK and PPM in multipath channels and in the presence of synchronization errors. We will use CM1, CM3, and CM4 of IEEE 802.15.4a [22] in simulation.

Figures 9 and 10 show the BER performance comparisons of GFSK and PPM in multipath channels. The CM4 model is used in simulation. Synchronization is perfect, and the maximum channel spread $D$ is truncated to 80 ns. The frame length is designed using the method mentioned in Section 4, so IFI is avoided in simulation. In this article, $\delta=T_{0}=T_{1}$ for PPM, and the $T_{0}$ of GFSK equals the $T_{0}$ of PPM. In the following, when a value of $\delta$ is given, it implies that $T_{0}$ and $T_{1}$ also have the same value. The analytical BER curves of PPM and GFSK are obtained directly from (24) and (28), respectively. In these two equations, we need to know

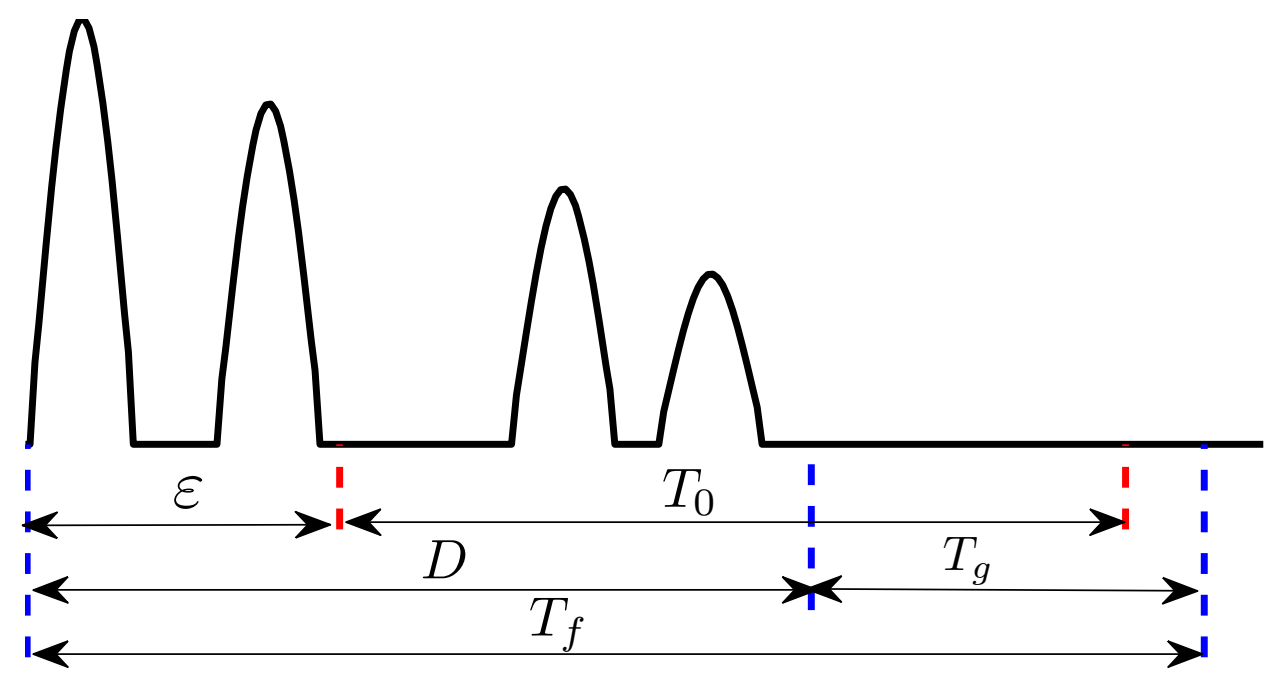

Figure 7 A GFSK frame structure in the presence of synchronization errors. 


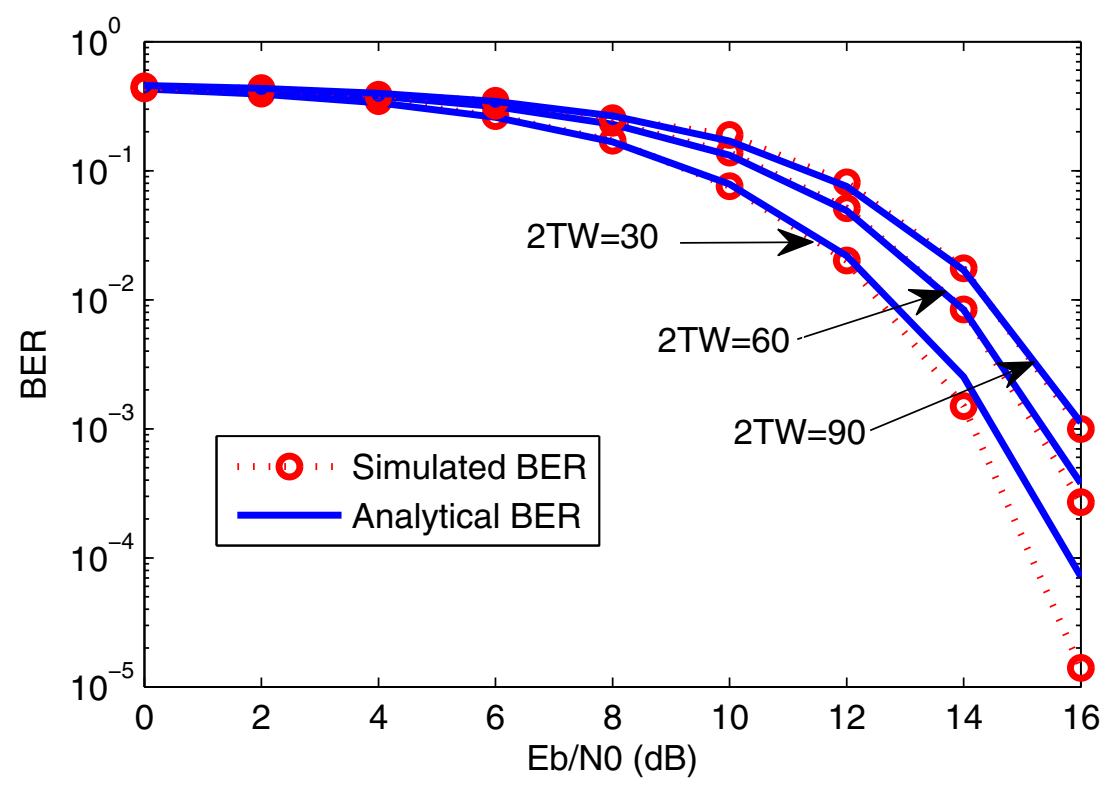

Figure 8 BER performance of GFSK for different 2TW values in AWGN channels

the values of parameter $\beta_{a}, \beta_{b}$ and $\lambda$. There is no mathematical formula to calculate the captured energy as a function of the length of the integration interval for IEEE 802.15.4a channel. We use a statistic method to obtain values for the above parameters. Firstly, we use the MATLAB code in [22] to generate realizations of the channel impulse response $h(t)$. Then we calculate the ratio of energy in a specific time interval to the total energy of a channel realization to obtain values for these parameters. These values are substituted into (24) and (28) to achieve the analytical BER. Both the simulated and the analytical BER are obtained by averaging over 100 channel realizations. In Figure 9, when $\delta=80$ ns, no CMI occurs and GFSK and PPM obtain the same BER. The analytical curves of GFSK and PPM match very well, as do the simulated curves. When $\delta=50 \mathrm{ns,}$ GFSK obtains better BER performance than PPM, and the improvement is approximately $0.2 \mathrm{~dB}$ at $\mathrm{BER}=10^{-3}$.

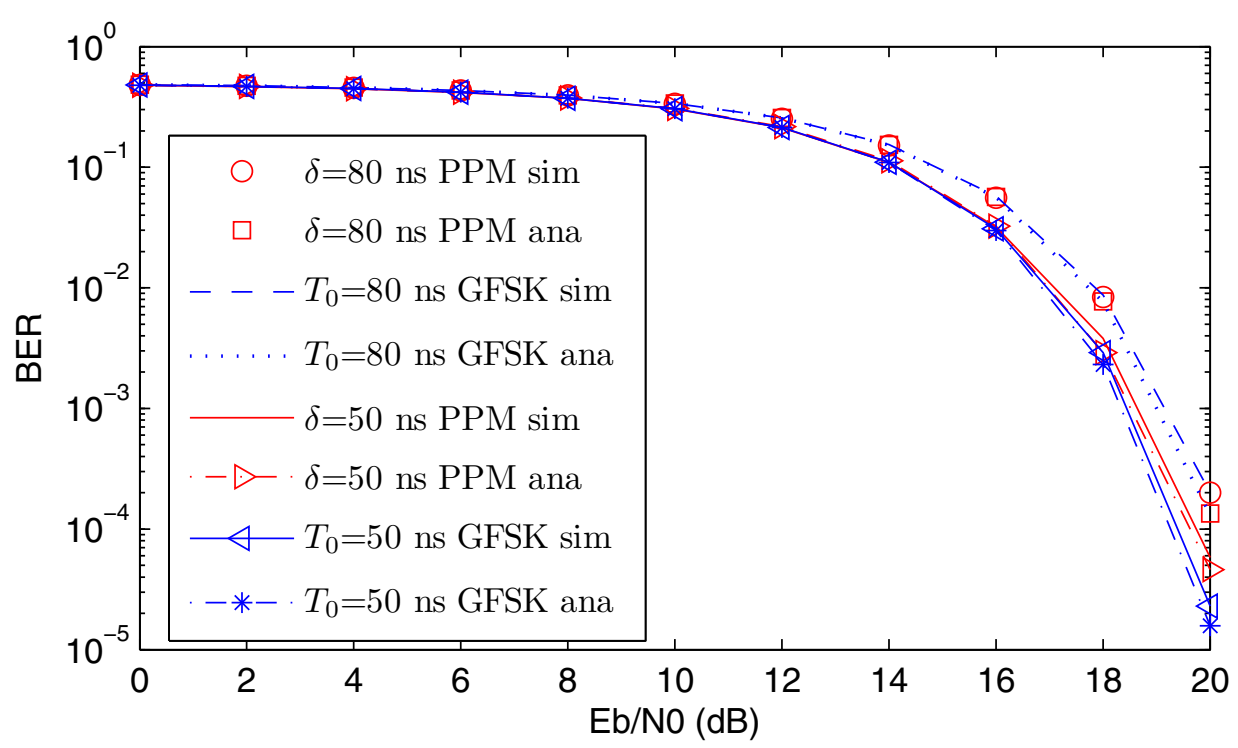

Figure 9 Comparisons of BER performance of GFSK and PPM in multipath channels (CM4 model, $D=80 \mathrm{~ns}, \delta=80$ and $50 \mathrm{~ns}$ ). 


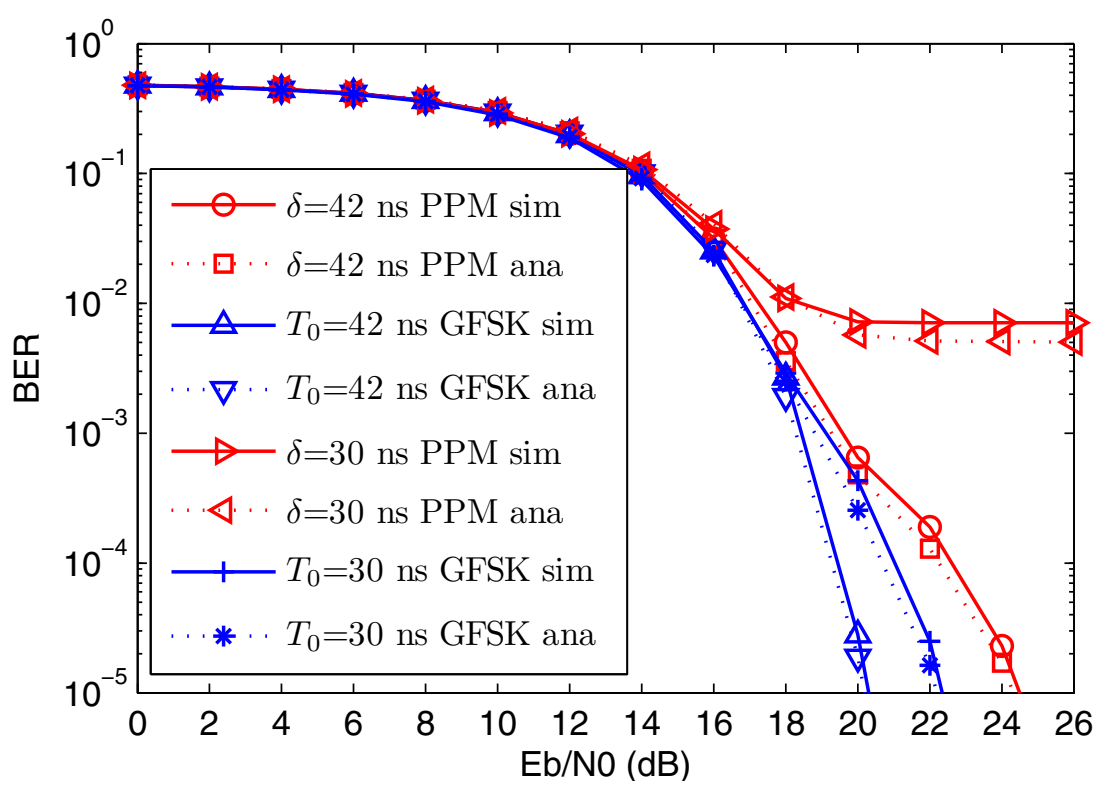

Figure 10 Comparisons of BER performance of GFSK and PPM in multipath channels (CM4 model, $D=80 \mathrm{~ns}, \delta=42$ and $30 \mathrm{~ns}$ ).

The reason is that $\delta$ is less than $D$, CMI occurs, and PPM performance is degraded. However, we can see from Figure 9 that the performances of GFSK and PPM are improved compared to when $\delta=80$ ns. This phenomenon can be explained as follows. The multipath components existing in the time interval between 50 and $80 \mathrm{~ns}$ include low signal energy, so the integrators capture more noise energy than signal energy in this interval. In Figure 10, when $\delta=42$ ns, GFSK obtains approximately $1.2 \mathrm{~dB}$ improvement at $\mathrm{BER}=10^{-3}$. When $\delta=30 \mathrm{~ns}$, GFSK requires an increase of $E_{b} / N_{0}$ approximately $0.7 \mathrm{~dB}$ to maintain $\mathrm{BER}=10^{-3}$, but PPM can not achieve this BER level and exhibit a BER floor. The BER performance of PPM cannot be improved by increasing the signal transmitting power. The reason is that when the signal power is increased, CMI is increased proportionally [23]. Unlike PPM, however, GFSK still achieves a good BER performance when the signal transmitting power is increased.

Figures 11 and 12 show the comparisons of BER performance when synchronization errors occur. In simulation, the modulation index $\delta$ is set to the maximum channel spread $D=80 \mathrm{~ns}$, so no CMI is in simulation. The frame structure is designed by following the method mentioned in Section 5, so IFI is avoided in simulation. The analytical BER curves are obtained directly from (31) and (35), and the values for parameters $\eta$ and $\rho$ in (31) and (35) are obtained using the statistic method similar to the one described above. Both the simulated and analytical BERs are obtained by averaging over 100 channel realizations. In Figure 11, when $\varepsilon=0 \mathrm{~ns}$, no synchronization error occurs, and GFSK and PPM achieve the same BER performance. When $\varepsilon=2 \mathrm{~ns}$, GFSK has better BER performance than PPM. The improvement at BER $=10^{-3}$ is approximately $1 \mathrm{~dB}$. In Figure 12, when $\varepsilon=3$ ns, GFSK obtains approximately $2.5 \mathrm{~dB}$ improvement at $\mathrm{BER}=10^{-3}$. When $\varepsilon=10 \mathrm{~ns}$, the BER of PPM is extremely bad and exhibits a BER floor because of severe synchronization errors, but GFSK still achieves a good BER.

In Figures 13, 14, 15, and 16, the BER performances of GFSK and PPM are compared in CM1 model. The maximum channel spread of CM1 model is truncated to 80 ns. In Figures 13 and 14, the comparisons of BER performance in multipath channels are shown. In Figure 13 , the $\delta$ values are 80 and $55 \mathrm{~ns}$, respectively. When $\delta$ $=80 \mathrm{~ns}$, GFSK and PPM achieve the same BER performance. When $\delta=55 \mathrm{~ns}$, GFSK achieves approximately $0.6 \mathrm{~dB}$ improvement at $\mathrm{BER}=10^{-3}$. In Figure 14, when $\delta=53 \mathrm{~ns}$, GFSK achieves approximately $6.2 \mathrm{~dB}$ improvement at BER $=10^{-3}$. When $\delta=50 \mathrm{~ns}$, GFSK still achieves a good BER performance. However, PPM exhibits a BER floor. Figures 15 and 16 show the comparison of BER performance in the presence of synchronization errors. In Figure 15, when $\varepsilon=0$ ns, GFSK and PPM achieve the same BER performance. When $\varepsilon=0.1 \mathrm{~ns}$, PPM has already exhibited a BER floor. In Figure $16, \varepsilon=0.5$ and $1 \mathrm{~ns}$, respectively. The BER curves of PPM all exhibit BER floors. However, GFSK still achieves good BER performance. In CM1 model, there exists a line of sight (LOS) component, and it includes great energy of the signal. A small 


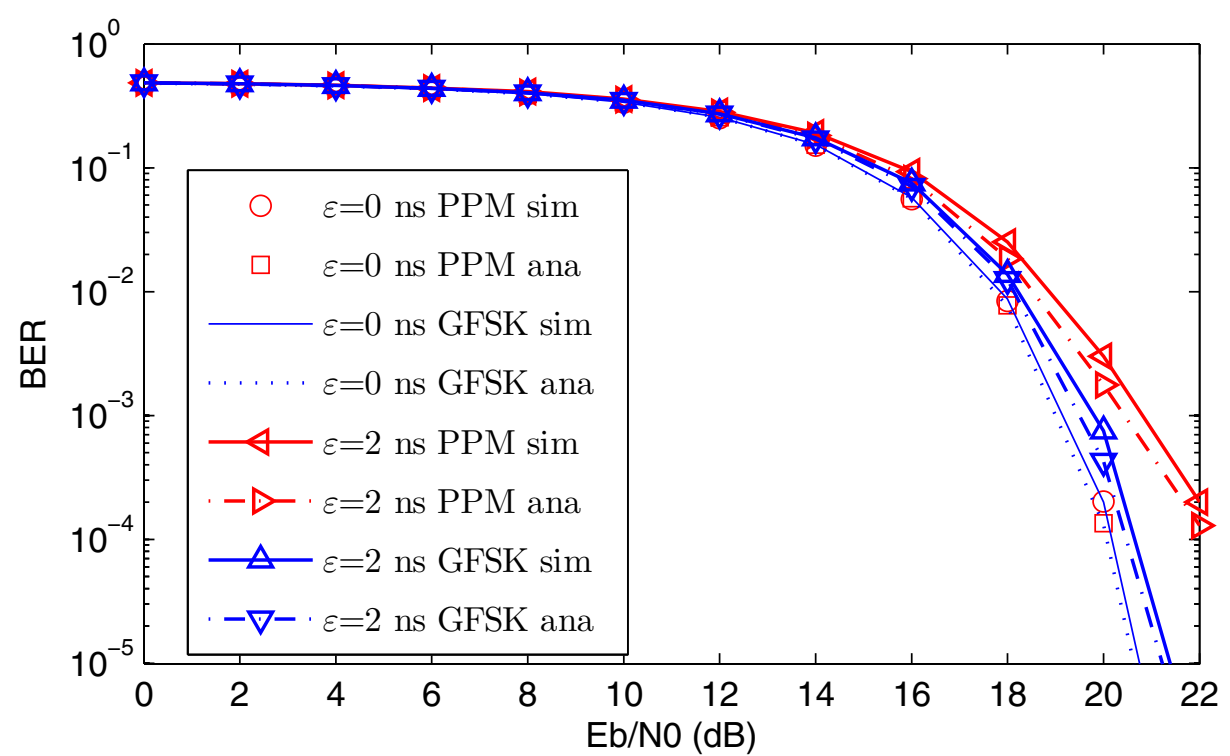

Figure 11 Comparisons of BER performance of GFSK and PPM in the presence of synchronization errors (CM4 model, $\delta=D=80 \mathrm{~ns}, \varepsilon$ $=0$ and $2 \mathrm{~ns}$ ).

synchronization error also can lead to a great performance degradation of PPM, since the signal energy of LOS component falls into wrong integration interval. In Figures 17, 18, 19, and 20, the BER performance of GFSK and PPM are compared in CM3 model. The maximum channel spread of CM3 is truncated to $80 \mathrm{~ns}$. In Figures 17 and 18, the comparisons of BER performance in multipath channels are shown. In Figure 17, when $\delta$ $=80 \mathrm{~ns}$, GFSK and PPM achieve the same BER performance. When $\delta=44 \mathrm{~ns}$, GFSK achieves $1.7 \mathrm{~dB}$ improvement at BER $=10^{-3}$. In Figure 18, when $\delta=20 \mathrm{ns,}$ GFSK achieves $3.7 \mathrm{~dB}$ improvement at $\mathrm{BER}=10^{-3}$. When $\delta=15 \mathrm{~ns}$, GFSK only needs an increase of $0.4 \mathrm{~dB}$ to maintain $\mathrm{BER}=10^{-3}$. However, PPM exhibits a BER

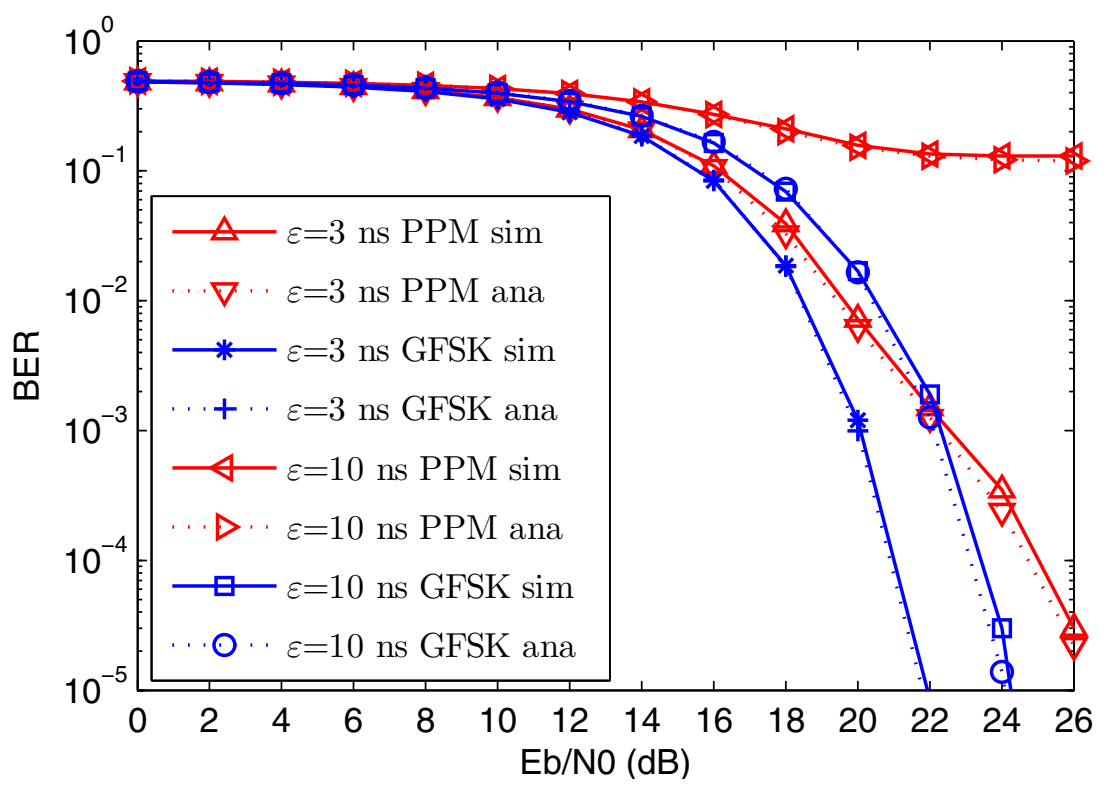

Figure 12 Comparisons of BER performance of GFSK and PPM in the presence of synchronization errors (CM4 model, $\delta=D=80 \mathrm{~ns}, \varepsilon$ $=3$ and $10 \mathrm{~ns}$ ). 


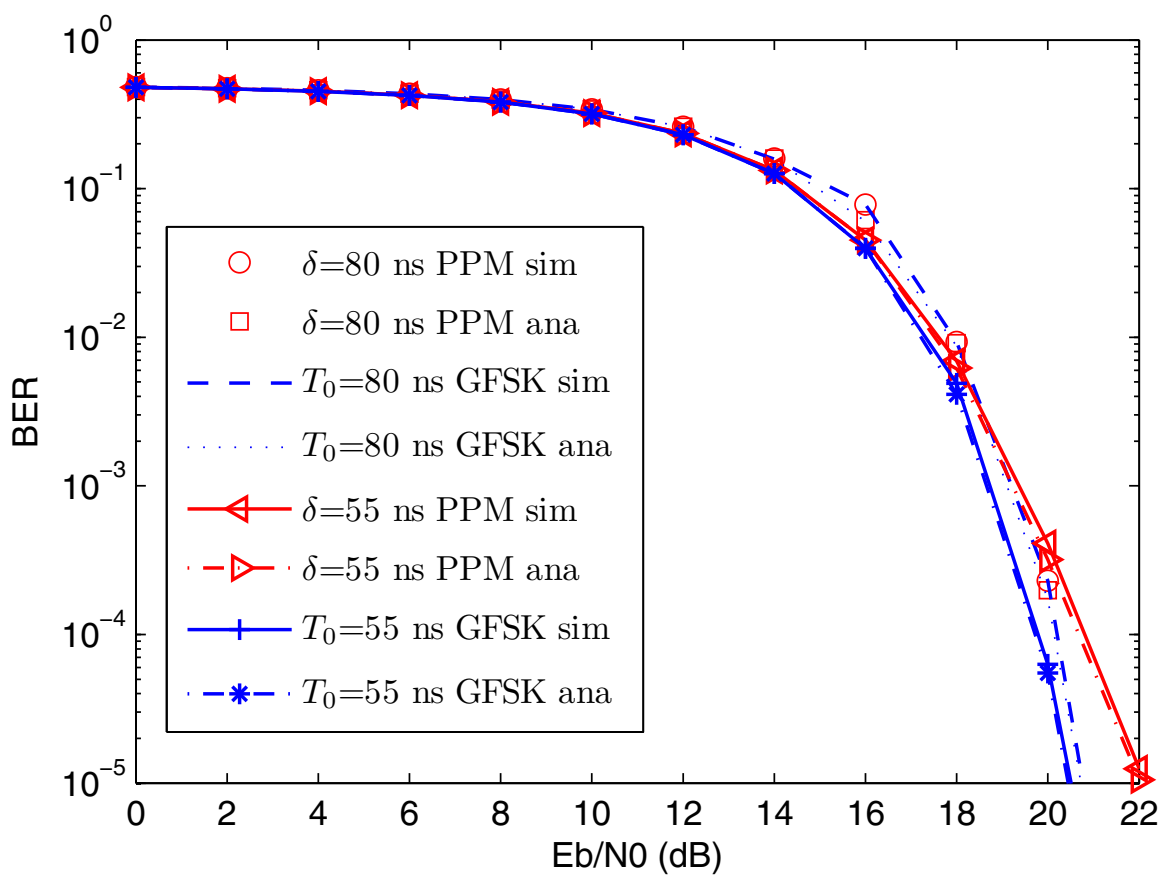

Figure 13 Comparisons of BER performance of GFSK and PPM in multipath channels (CM1 model, $D=80 \mathrm{~ns}, \delta=80$ and $55 \mathrm{ns)}$.

floor. In Figures 19 and 20, the BER performances are compared in the presence of synchronization errors. In Figure 19, when $\varepsilon=0$ ns, GSFK and PPM achieve the same BER performance. When $\varepsilon=0.05 \mathrm{~ns}$, GFSK achieves approximately $1.5 \mathrm{~dB}$ improvement at $\mathrm{BER}=$ $10^{-3}$. In Figure $20, \varepsilon=0.1$ and $0.2 \mathrm{~ns}$, respectively. The BER curves of PPM both exhibit BER floors. However, GFSK still achieves good BER performance. CM3 model

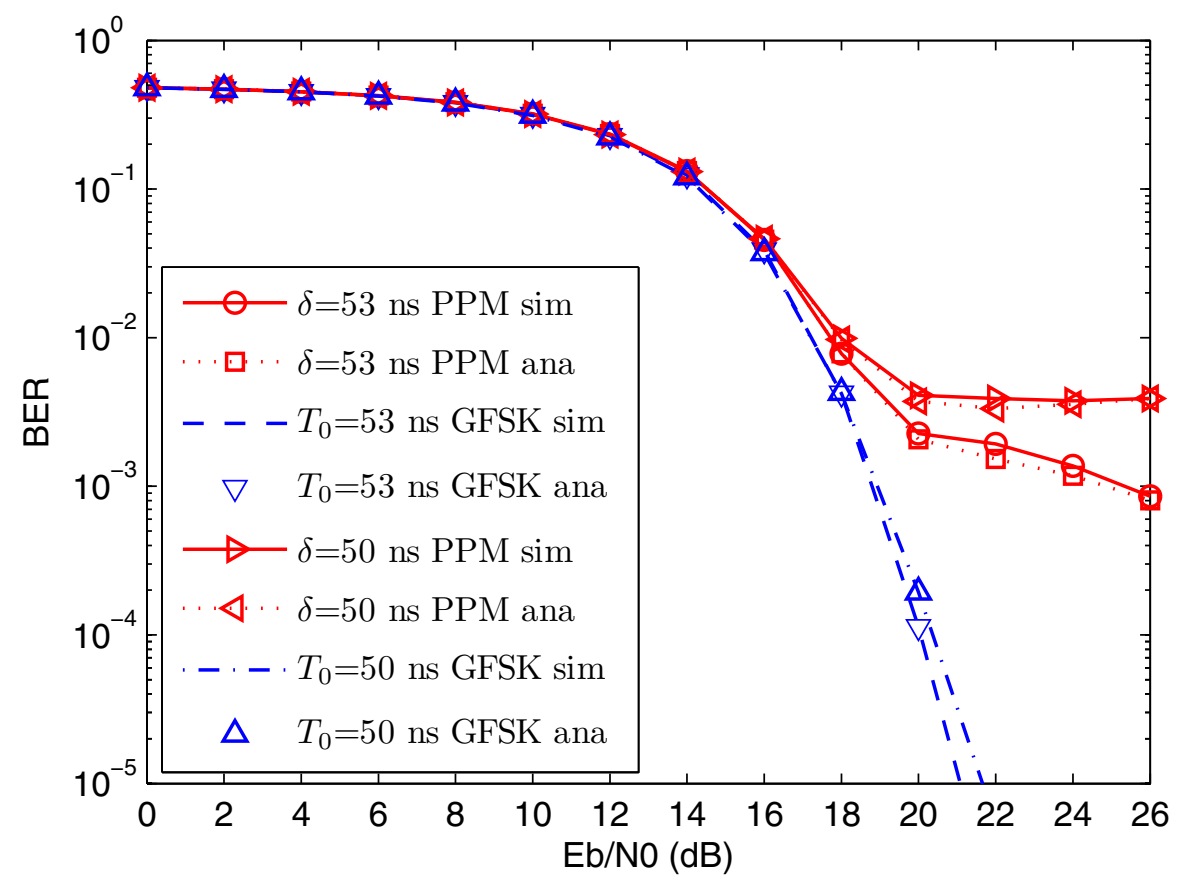

Figure 14 Comparisons of BER performance of GFSK and PPM in multipath channels (CM1 model, $D=80 \mathrm{~ns}, \delta=53$ and $50 \mathrm{~ns}$ ). 


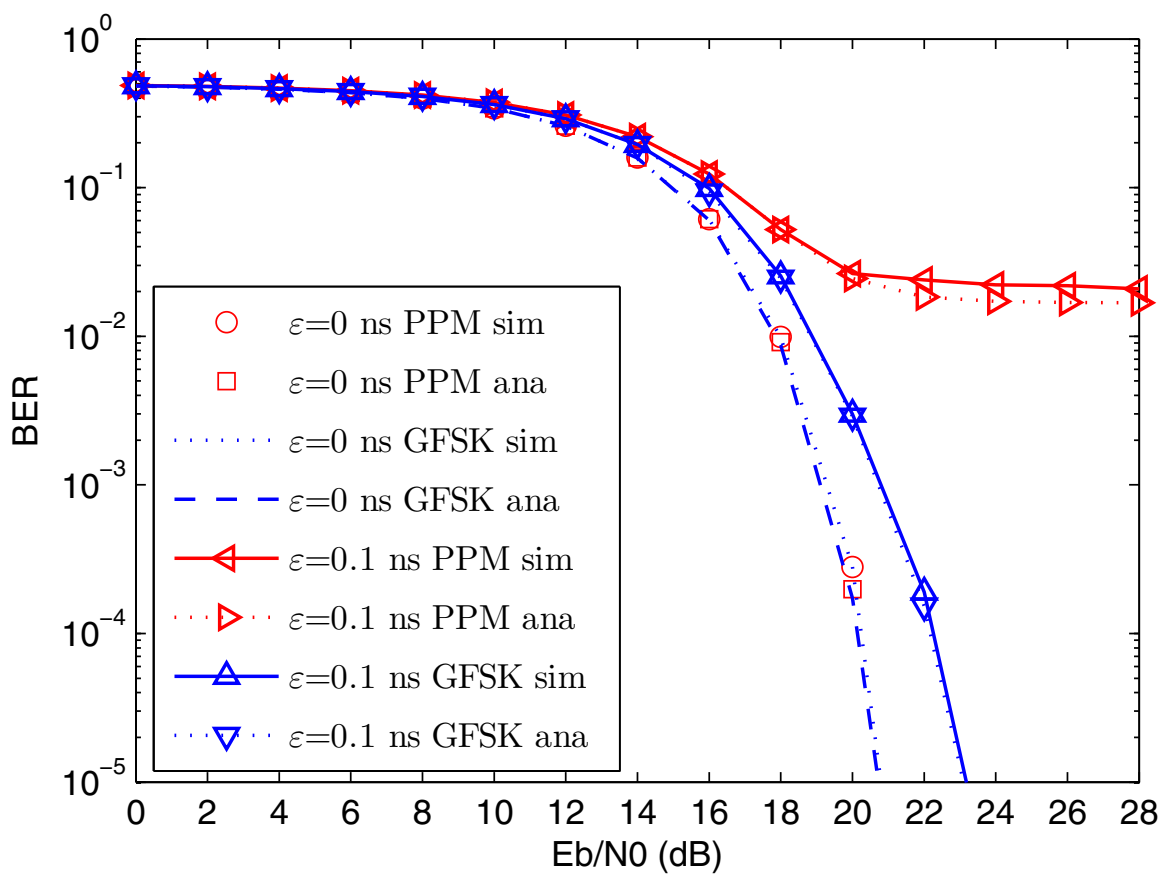

Figure 15 Comparisons of BER performance of GFSK and PPM in the presence of synchronization errors (CM1 model, $\delta=D=80$ ns, $\varepsilon$ $=0$ and $0.1 \mathrm{~ns}$ ).

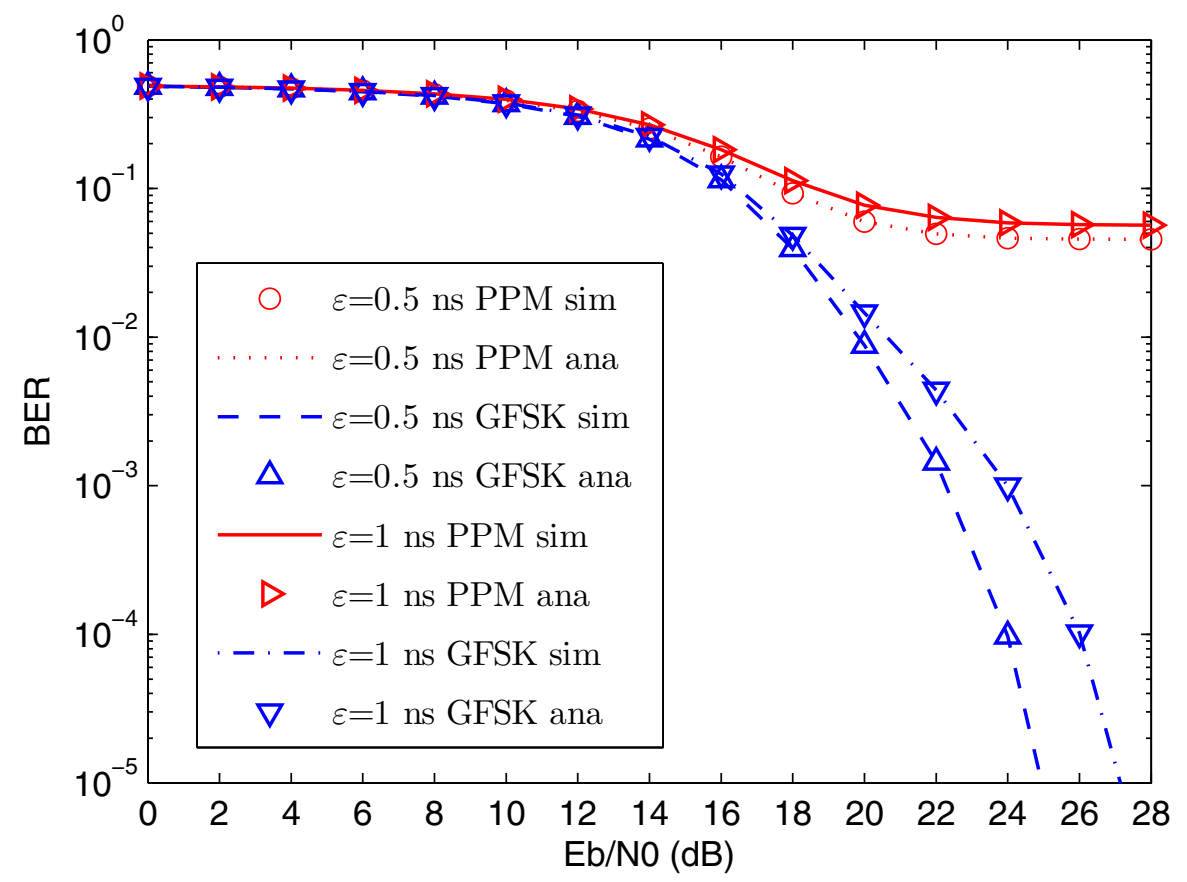

Figure 16 Comparisons of BER performance of GFSK and PPM in the presence of synchronization errors (CM1 model, $\delta=D=80 \mathrm{~ns}, \varepsilon$ $=0.5$ and $1 \mathrm{~ns}$ ). 


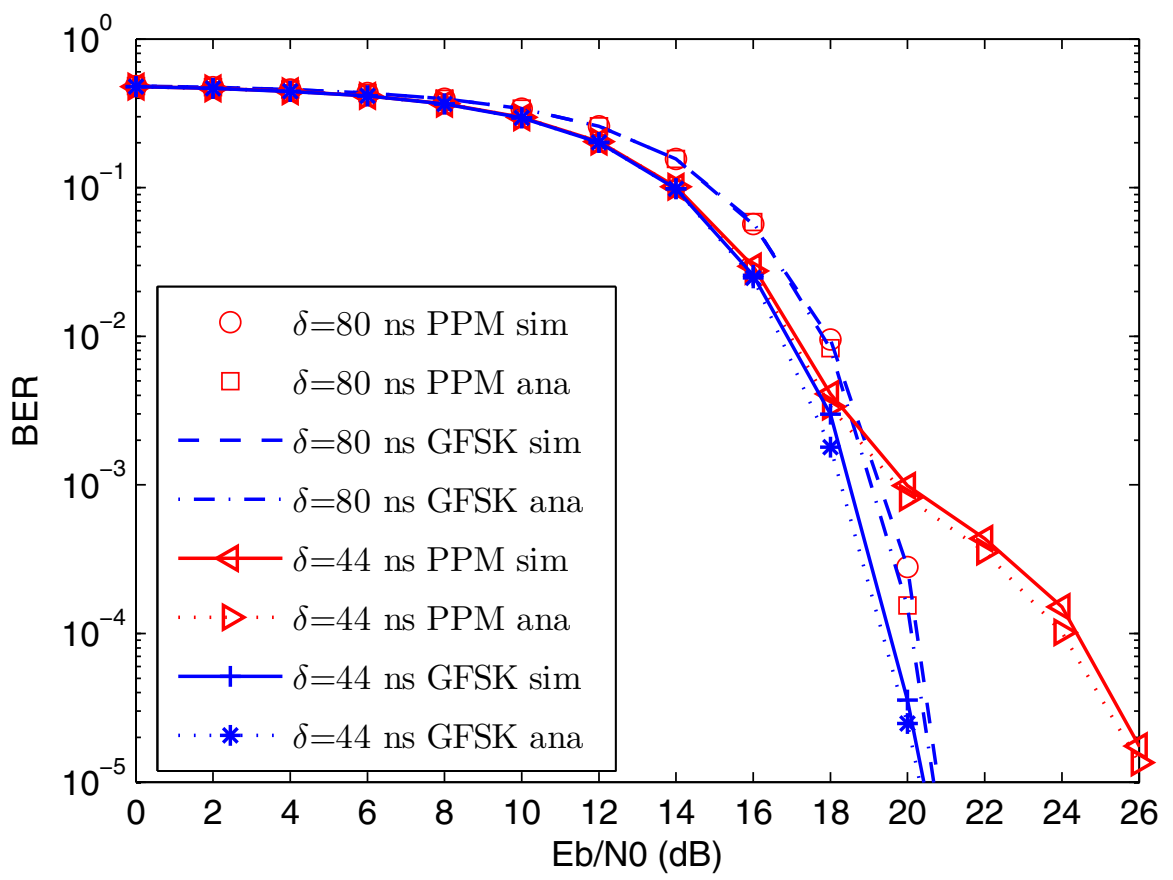

Figure 17 Comparisons of BER performance of GFSK and PPM in multipath channels (CM3 model, $D=80 \mathrm{~ns}, \delta=80$ and $44 \mathrm{ns)}$.

also includes an LOS component, and PPM is very sensitive to synchronization errors in CM3. In a PPM system, modulation is achieved by shifting the pulse position, and the orthogonality of the signals is achieved in time domain. When CMI or synchronization errors occur, this orthogonality is easily destroyed. The orthogonality of a GFSK system is achieved in the frequency domain. Although the integration interval and

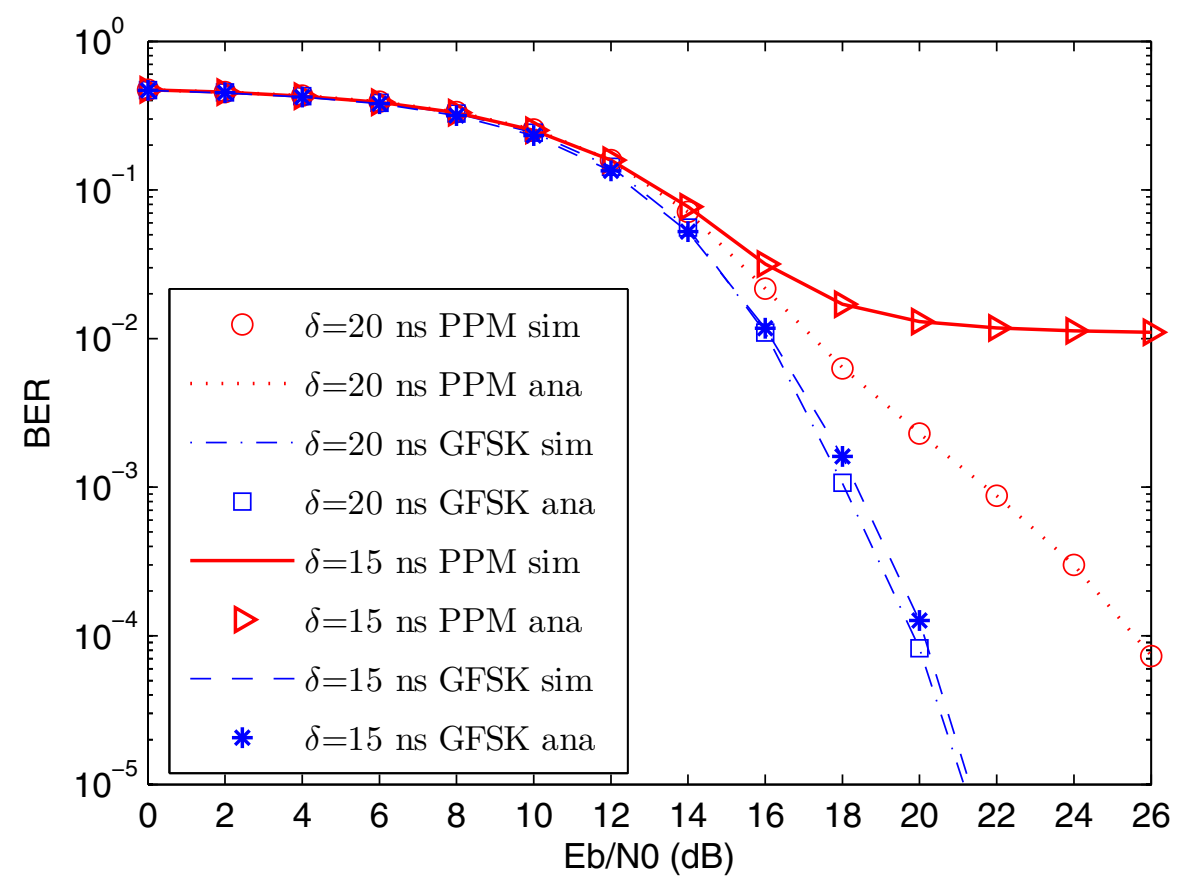

Figure 18 Comparisons of BER performance of GFSK and PPM in multipath channels (CM3 model, $D=80 \mathrm{~ns}, \delta=20$ and $15 \mathrm{~ns}$ ) 


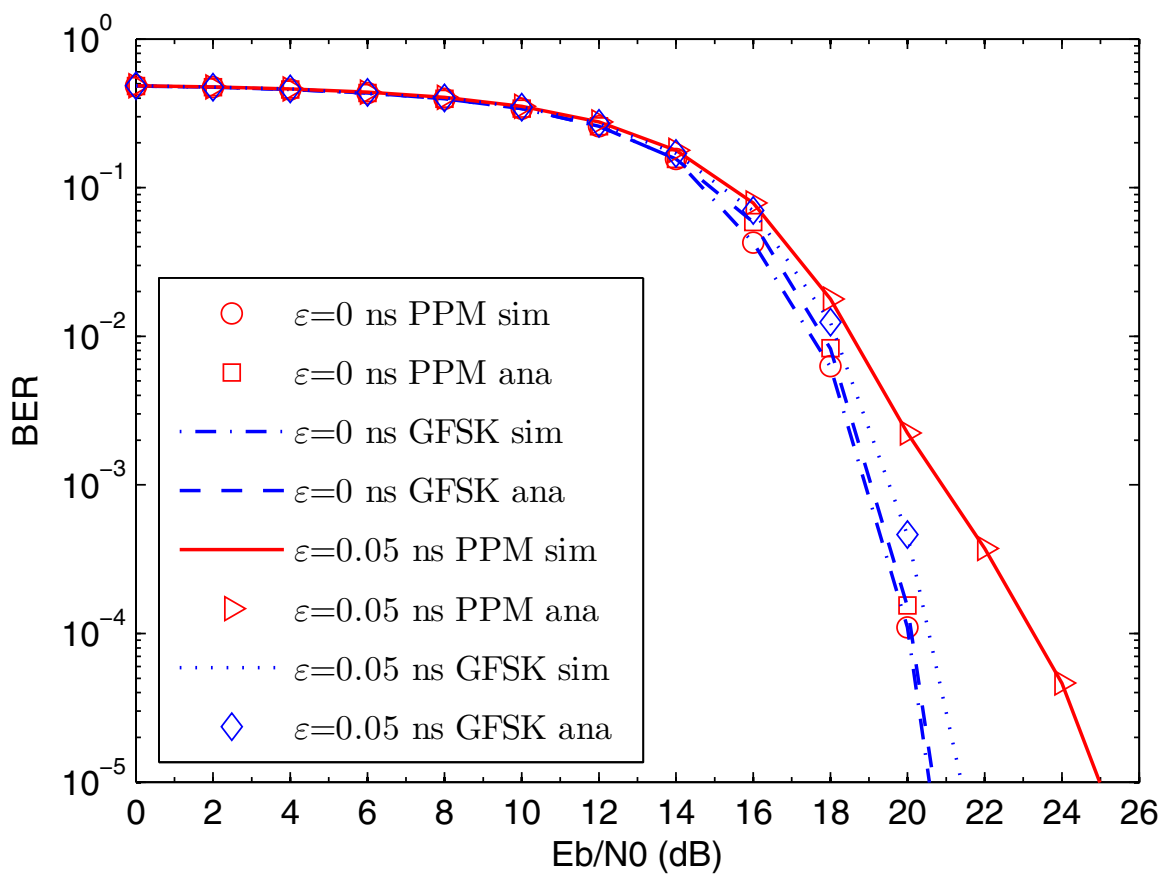

Figure 19 Comparisons of BER performance of GFSK and PPM in the presence of synchronization errors (CM3 model, $\delta=D=80 \mathrm{~ns}, \varepsilon$ $=0$ and $0.05 \mathrm{~ns}$ ).

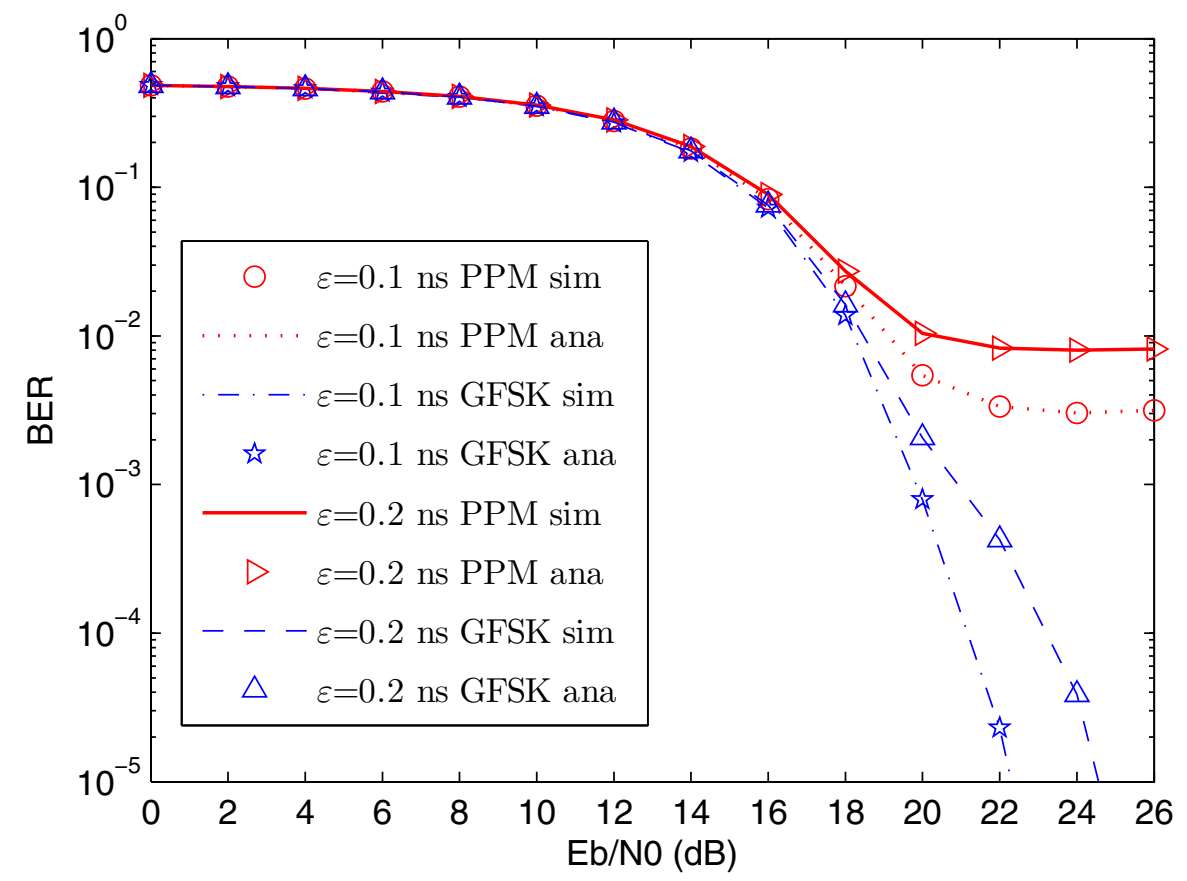

Figure 20 Comparisons of BER performance of GFSK and PPM in the presence of synchronization errors (CM3 model, $\delta=D=80 \mathrm{~ns}, \varepsilon$ $=0.1$ and $0.2 \mathrm{~ns}$ ) 
synchronization error also affect performance of a GFSK system, its orthogonality is not affected by these two factors. This is why a GFSK system has better BER performance than a PPM system in the presence of CMI and synchronization errors. Since the usable frequency is constrained by many possible institutional regulations, such as FCC emission mask, we cannot enlarge the signal bandwidth to infinity. The maximum possible signal bandwidth of a single pulse in a GFSK system is at most one half of that of a PPM system. But this does not mean that the maximum possible data rate of a GFSK system is one half of that of a PPM system. In UWB channels, the multipath components are resolvable and not overlapped due to the extremely short pulse duration. And each pulse will generate many multipath components and the arriving time of each multipath component is not decided by the pulse but the channel environment. Usually, the maximum channel spread $D$ is very long when compared to a single pulse duration. Although the single pulse duration of a GFSK system is twice that of a PPM system, but the values of $D$ are almost the same. Because the multipath components in these two system arrive at the same time and the only difference is the duration of the pulses in these two systems. But the difference of the durations of the pulses in these two systems is very small when compared to maximum channel spread. If we chose the value of $D$ from either GFSK or PPM systems as a common reference value, the signal energies of these two systems in the time interval $[0, D]$ will be almost the same.

The tiny difference is no more than half of the energy of the last multipath component in this range. Usually, this multipath component includes very low signal energy, so the energy difference can be neglected. So we can obtain the same maximum channel spread for GFSK and PPM systems despite the pulse duration of a GFSK system is twice that of a PPM system. We also verify our conclusion using the Matlab code in [22] and these two systems both obtain the same values of $D=$ 80 ns. However, the frame of a PPM system include two intervals, $T_{0}$ and $T_{1}$, so its frame period is twice that of a GFSK system. This leads to the data rate in a PPM system will be half of that of a GFSK system.

Since GFSK does not suffer from CMI as PPM, it is more suitable for high data rate UWB systems than PPM. From the above simulation, we can know that GFSK still achieves a good BER performance when we chose a $T_{0}$ value much smaller than maximum channel spread. However, PPM suffers from CMI, so the BER performance is considerably worse when $\delta$ is smaller than maximum channel spread. The computation costs of GFSK and PPM are almost the same. PPM performs integration over two time intervals $T_{0}$ and $T_{1}$ and then subtracts the two outcomes from the integrator to generate the decision variable. GFSK performs integration over two branches and subtracts the two outcomes from two integrators to generate the decision variable. The computation costs of these two systems are in the same rank. The difference is that GFSK needs two pulse generators at the transmitter and two branches at the receiver. However, this does not increase the complexity of GFSK too much. As mentioned above, many methods to generate different-order derivatives of the Gaussian pulse have been proposed and the cost of using two pulse generators is not expensive. Other components at the transmitter can be shared by these two pulse generators, such as the power amplifier and other baseband components. At the receiver side, the system needs two ED receiver branches which have filters with different frequency range. ED receiver has been a very mature technology for many years and the structure of the receiver is simple and easy to implement. Two branches in GFSK system do not increase the complexity of the receiver too much. One just adds another simple branch to the receiver and the cost is low. Especially, when the system uses digital receiver, the current semiconductor industry uses FPGA or ASIC to build the whole system on a single chip at a very low price. The hardware engineer only need to write computer program to implement the system by Verilog or VHDL language. The two branches of GFSK systems only need to create two instances of the single branch. And it will not occupy too much chip space. Usually the chip has much more redundant space than the actual requirement of the system and the additional branch just occupies the redundant space. We also can see many similar examples about two branches receiver, such as noncoherent receiver of conventional carrier-based FSK system. The complexity is not a problem in either these systems or our system.

The above analysis does not consider the possible effect of narrow band interference to our GFSK system. Narrow band interference will change the energy of signal spectra and lead to the unbalanced energy of pulse for bits 0 and 1 . This can be resolved by using notch filter. There are many mature methods about using notch filter to mitigate the narrow band interference in UWB systems [24-30]. The system can transmit training sequence including both bits 0 and 1 , and the training sequence is known by both transmitter and receiver. The receiver can detect the spectrum of interference signal by comparing the spectrum of received signal with a predefined pulse spectrum. If the interference signal is detected, the adaptive notch filter will work and adjust its coefficients to mitigate the spectrum of interference. Finally, the composite spectrum of received signal and interference signal is like the spectrum of the pulse we want. The above procedure will be performed in both 
frequency ranges of pulses for bits 0 and 1 . After the application of the notch filter, we still can maintain the same energy for bits 0 and 1 at the receiver side, so the equations we derived above are still valid.

\section{Conclusion}

A new method GFSK to realize ED UWB system is proposed and this new method achieves the same BER performance as PPM in AWGN channels. However, after the signals pass through multipath channels, GFSK achieves better performance than PPM because it is not affected by CMI. Also when synchronization errors occur, GFSK achieves better BER performance than PPM. When these two methods occupy the same spectral width, GFSK can achieve higher data rate than PPM.

\section{Competing interests}

The authors declare that they have no competing interests.

Received: 31 August 2011 Accepted: 19 December 2011

Published: 19 December 2011

\section{References}

1. MZ Win, RA Scholtz, Impulse radio: how it works. IEEE Commun Lett. 2(2), 36-38 (1998). doi:10.1109/4234.660796

2. N Zhang, T Zhang, Q Zhang, A study on performance of an IR-UWB receiver based on energy detection. in IEEE International Conference on WiCOM, Dalian, China 1-5 (2008)

3. $\mathrm{N} \mathrm{He}, \mathrm{C}$ Tepedelenlioglu, Performance analysis of non-coherent UWB receiver at different synchronization levels. IEEE Trans Wirel Commun. 5(6), 1266-1273 (2006)

4. D Mu, Z Qiu, Weighted non-coherent energy detection receiver for UWB OOK systems. in Proceedings of the IEEE 9th ICSP, Beijing, China 1846-1849 (2008)

5. S Dubouloz, B Denis, S Rivaz, L Ouvry, Performance analysis of LDR UWB non-coherent receivers in multipath environments. in IEEE ICU'05, Zurich, Switzerland 491-496 (2005)

6. NK Askar, SC Lin, HD Pfister, GE Rogerson, DS Furuno, Spectral keying: a novel modulation scheme for UWB systems. in IEEE Conference on U/tra Wideband Systems and Technologies, Reston, Virginia, USA 418-422 (2003)

7. JFM Gerrits, JR Farserotu, Ultra wide band FM: a constant envelope frequency domain approach. in IEEE 2004 International Zurich Seminar on Communications, Zurich, Switzerland 90-93 (2004)

8. F Ramirez-Mireles, T Cooklev, GA Paredes-Orozco, UWB-FSK: performance tradeoffs for high and low complexity receivers. IEEE Trans Consum Electron. 56(4), 2123-2131 (2004)

9. F Nekoogar, Ultra-Wideband Communications: Fundamentals and Applications, (Prentice Hall, Upper Saddle River, NJ, 2005), p. 8.43

10. H Harada, K Ikemoto, R Kohno, Modulation and hopping using modified Hermite pulses for UWB communications. in IEEE International Workshop on Ultra Wideband Systems, Kyoto, Japan 336-340 (May 2004)

11. LB Michael, M Ghavami, R Kohno, Multiple pulse generator for ultrawideband communication using Hermite polynomial based orthogonal pulses, in IEEE Conference on Ultra Wideband Systems and Technologies, Wyndham Baltimore Inner Harbor, USA, pp. 47-51 (2002)

12. JA Silva, MLR Campos, Spectrally efficient UWB pulse shaping with application in orthogonal PSM. IEEE Trans Commun. 55(2), 313-322 (2007)

13. M Benedetto, G Giancola, Understanding Ultra Wide Band Radio Fundamentals, Prentice Hall, Upper Saddle River, NJ, p. 32. 187-202 (2004)

14. T Phan, V Krizhanovskii, S Han, S Lee, H Oh, N Kim, 4.7pJ/pulse 7th derivative Gaussian pulse generator for impulse radio UWB. IEEE Int Symp Circuits and Systems, New Orleans, USA 3043-3046 (2007)

15. D Kim, G Bang, C Park, Design and characteristics of high order derivative Gaussian pulse generator for DS-UWB. IEEE Asia-Pacific Microwawe Conference, Yokohama, Japan 1110-1113 (2006)
16. A Zadok, X Wu, J Sendowski, A Yariv, AE Willner, Reconfigurable generation of high-order ultra-wideband waveforms using edge detection. J Lightw Technol. 28(16), 2207-2212 (2010)

17. $X$ Cheng, $Y$ Guan, Mitigation of cross-modulation interference in UWB energy detector receiver. IEEE Commun Lett. 13(6), 375-377 (2009)

18. H Urkowitz, Energy detection of unknown deterministic signals. Proc IEEE. 55(4), 523-531 (1967)

19. PA Humblet, M Azizoglu, On the bit error rate of lightwave systems with optical amplifiers. J Lightw Technol. 9(11), 1576-1582 (1991). doi:10.1109/ 50.97649

20. H Celebi, H Arslan, Cross-modulation interference and mitigation technique for ultrawideband PPM signaling. IEEE Trans Veh Technol. 57(2), 847-858 (2008)

21. RF Mills, GE Prescott, A comparison of various radiometer detection models IEEE Trans Aerospace Electron Syst. 32(1), 467-473 (1996)

22. AF Molisch, K Balakrishnan, D Cassioli, C Chong, S Emami, A Fort, J Karedal, J Kunisch, H Schantz, U Schuster, K Siwiak, IEEE 802.15.4a channel modelfinal report. http://www.ieee802.org/15/pub/04/15-04-0662-02-004achannel-model-final-report-r1.pdf. Accessed June 2011

23. H Arslan, Cross-modulation interference reduction for pulse-position modulation UWB signals. in IEEE Veh Tech Conf., Montreal, Canada 1-5 (2006)

24. A Vallese, A Bevilacqua, C Sandner, M Tiebout, A Gerosa, A Neviani, Analysis and design of an integrated notch filter for the rejection of interference in UWB systems. IEEE J Solid State. 44(2), 331-343 (2009)

25. K Thirumalaivasan, R Nakkeeran, UWB bandpass filter with notched band for the rejection of $5 \mathrm{GHz}$ WLAN using hexagonal multiple mode resonator. in IEEE International Conference on Communication Control and Technologies, Ramanathapuram, India 21-24 (2010)

26. K Li, D Kurita, T Matsui, UWB bandpass filters with multi notched bands. in IEEE 36th European Microwave Conference, Manchester, UK 591-594 (2006)

27. Q Wei, H Wei, J Hong, J Wang, Design and implementation of UWB bandpass filter with a frequency notch for choking back the interference from narrow band wireless communication systems. in IEEE International Conference on Ultra-Wideband, Vancouver, Canada 521-524 (2009)

28. B Liu, Y Yin, Y Yang, Y Wei, A Sun, UWB bandpass filter with notched band using broadside-coupled microstrip-coplanar waveguide structure. in IEEE International Conference on Microwave Technology and Computational Electromagnetics, Beijing, China 247-249 (2011)

29. B Yao, Y Zhou, Q Cao, A UWB bandpass filter with multi notched bands using microstrip/coplanar waveguide. in IEEE 8th International Symposium on Antennas, Propagation and EM Theory, Kunming, China 637-640 (2008)

30. X Luo, H Qian, J Ma, K Ma, KS Yeo, A compact UWB bandpass filter with ultra narrow notched band and competitive attenuation slope. in IEEE International Microwave Symposium Digest, Anaheim, USA 221-224 (2010)

doi:10.1186/1687-1499-2011-206

Cite this article as: Cui and Xiong: UWB system based on energy detection of derivatives of the Gaussian pulse. EURASIP Journal on Wireless Communications and Networking 2011 2011:206.

\section{Submit your manuscript to a SpringerOpen ${ }^{\circ}$ journal and benefit from:}

- Convenient online submission

- Rigorous peer review

- Immediate publication on acceptance

- Open access: articles freely available online

- High visibility within the field

- Retaining the copyright to your article

Submit your next manuscript at $>$ springeropen.com 